\title{
Experimental assessment of bi-directional transmission distribution functions using digital imaging techniques
}

\author{
Marilyne Andersen ${ }^{1}$, Laurent Michel, Christian Roecker, Jean-Louis Scartezzini \\ Laboratoire d'Energie Solaire et de Physique du Bâtiment, Ecole Polytechnique \\ Fédérale de Lausanne, CH - 1015 Lausanne, Switzerland
}

\begin{abstract}
Many daylighting applications require a precise knowledge of the directional transmission features of advanced fenestration materials. These photometric properties are described by a bi-directional transmission distribution function (BTDF), whose experimental assessment requires an appropriate equipment.

A novel bi-directional photogoniometer, based on digital imaging techniques, has been designed and developed for that purpose. The main advantages of this device are the significant reduction of the time required for data measurement and its capability to assess an almost continuous BTDF function. These features can be achieved only through detailed and accurate calibration procedures of the bidirectional photogoniometer, which are described in this paper, together with digital image and data processing. Several experimental results, obtained for different fenestration materials, are used to illustrate the capabilities of this novel equipment.
\end{abstract}

\footnotetext{
${ }^{1}$ Corresponding author. Tel.: +41-21-693-45-51; Fax : +41-21-693-27-22; E-mail: Marilyne.Andersen@epfl.ch 
Keywords: daylighting, photometry, digital imagery (CCD), bi-directional transmission distribution function (BTDF).

\section{Introduction}

Daylight distribution inside buildings must be improved, especially into deep sidelong rooms, in order to reduce building energy consumption and ameliorate visual comfort [1] [2]. Advanced fenestration systems, which include daylight redirecting devices, novel solar blinds and advanced glazing materials can play a significant role in this field.

The propagation of the daylight flux into building rooms can only be assessed, however, through the full knowledge of the bi-directional photometric properties of these fenestration materials. These features allow, already at the project's level, to define the judicious combination of glazing materials, which would be appropriate to a given lighting situation and aiming at better visual comfort and energy savings [3]. Daylighting simulation programs require also such detailed photometric data to achieve a reliable modelling of light propagation into rooms using advanced fenestration systems [4].

Accurate and objective measurements of the photometric properties of advanced glazings, characterised by a bi-directional transmission distribution function (BTDF), have therefore to be handled to control the daylighting performances of buildings. This function, also called $\mathrm{q}$ in the CIE nomenclature [5], is defined by equation (1), and the associated photometric and geometric quantities are illustrated in Fig. 1: 


$$
\operatorname{BTDF}\left(\theta_{1}, \phi_{1}, \theta_{2}, \phi_{2}\right)=\frac{L_{2}\left(\theta_{1}, \phi_{1}, \theta_{2}, \phi_{2}\right)}{L_{1}\left(\theta_{1}, \phi_{1}\right) \cos \theta_{1} d \omega_{1}}=\frac{L_{2}\left(\theta_{1}, \phi_{1}, \theta_{2}, \phi_{2}\right)}{E_{1}\left(\theta_{1}\right)}\left[\frac{c d}{m^{2} l x}\right]
$$

where

$\left(\theta_{1}, \phi_{1}\right)$ : Polar co-ordinates of incoming light flux $\left[{ }^{\circ}\right]$

$\left(\theta_{2}, \phi_{2}\right)$ : Polar co-ordinates of emerging (transmitted) light flux [ $\left.{ }^{\circ}\right]$

$\mathrm{L}_{1}\left(\theta_{1}, \phi_{1}\right)$ : Luminance of element of incoming light flux [cd m$\left.{ }^{-2}\right]$

$\mathrm{L}_{2}\left(\theta_{1}, \phi_{1}, \theta_{2}, \phi_{2}\right)$ : Luminance of emerging (transmitted) element of light flux [cd m${ }^{-2}$ ]

$\mathrm{d} \omega_{1}$ : Solid angle subtended by incoming light flux [sr]

$\mathrm{E}_{1}\left(\theta_{1}\right)$ : Illuminance of the fenestration material, due to the incoming light flux [lx]

Fig. 1. Photometric and geometric quantities used for BTDF definition.

Bi-directional photogoniometric devices have recently been developed to experimentally assess BTDFs $[6,7,8,9,10]$. Their conventional measurement principle consists of a movable photometric sensor measuring illuminances in different directions $\left(\theta_{2}, \phi_{2}\right)$, which leads to the associated transmitted luminances $\mathrm{L}_{2}\left(\theta_{1}, \phi_{1}, \theta_{2}, \phi_{2}\right)$. Even if showing accurate and reliable performances, the photometric devices based on this conventional method show two significant drawbacks:

- they are time-consuming, due to the required movements of the mobile photosensor

- they need a further refinement of the $\left(\theta_{2}, \phi_{2}\right)$ polar co-ordinates resolution, in case of a glazing material showing high luminance peaks of transmission [6].

To overcome these difficulties, a novel type of bi-directional photogoniometer has been designed, using advanced digital imaging techniques [11]. The experimental 
BTDF assessing procedures, together with the important effort of calibration of the equipment, are given in this paper. Several results of BTDF measurements are presented to illustrate the accuracy and capability of the photogoniometer.

\section{Methodology}

\subsection{Equipment description}

The photogoniometer's concept has been described extensively in [11], where the first strategies of calibration and image processing have been developed; Fig. 2 shows an illustration of the device.

Fig. 2. Bi-directional photogoniometer developed at LESO-PB/EPFL. The CCD camera (absent on the picture) is fixed on the rotating ring at the cross mark.

The general working principles of the bi-directional photogoniometer are the following:

- the equipment is placed underneath a calibrated light source (2.5 KW HMI discharge lamp), placed 6 meters above the main platform

- the rotation of the main platform (i.e. $\theta_{1}$ moving from $0^{\circ}$ to $90^{\circ}$ ) and the sample holder (i.e.: $\phi_{1}$ moving from $0^{\circ}$ to $360^{\circ}$ ) determine the incident beam direction (cf. Fig. 3)

- a CCD video camera and a triangular projection screen (covering $60^{\circ}$ in azimuth), towards which the camera is aiming, perform up to a $360^{\circ}$ rotation on a ring located underneath the main platform (cf. Fig. 2). 
Fig. 3. Rotation of main platform and sample holder.

The six screen positions, represented on Fig. 4, lead to the visualisation of the whole ( $2 \pi)$ steradian hemisphere of the transmitted rays, without any disturbances of measurements due to inter-reflections; all other internal elements are of highly absorbing material. A conic cap is fixed on the main platform (around the camera and the screen) to avoid parasitic light. The measurements are performed in a $5 \mathrm{~m} \mathrm{x}$ $5 \mathrm{~m}$ x 8m dark room to allow appropriate experimental conditions.

Fig. 4. Rotation of lambertian screen (orthogonal projection on the sample plane).

The CCD camera (Kappa CF 8/1 DXCair), providing 752 x 582 pixels images, is computer-controlled through a PC computer (Intel Pentium II $300 \mathrm{MHz}$ ) and appropriate digital images acquisition and handling software (IMAGE-PRO PLUS $\AA^{2}$ ). The latter offers integration intervals comprised between $100 \mu$ s and several hours; the lowest integration time interval used for the BTDFs assessment is $40 \mathrm{~ms}$, to avoid beating effects with the own pulsations of the light source (50 Hz AC supply). The CCD camera diaphragm aperture is fixed and manually set. The camera is calibrated on a grey scale, in order to be used as a multiple points luminance-meter; the projection screen is covered with a spectrally neutral diffusing white paint (LMT photometer paint).

The sample holder allows free sample sizes, with maximal dimensions of $40 \mathrm{~cm} \mathrm{x}$ $40 \mathrm{~cm}$. A set of diaphragms is used to limit the illuminated area of the sample to 10 , 17, 24, and $30 \mathrm{~cm}$ diameter; sample size and angular resolution are inversely 
proportional, as a consequence of a growing approximation in the outgoing direction for larger samples.

\subsection{Calibration procedures}

The main calibration procedures to achieve concern the CCD video camera, used as a fast and accurate luminance-meter. The following calibrations must thus be performed:

Spectral calibration:

- $\quad$ The CCD video camera must be calibrated spectrally, that is, its spectral response has to be as close to the human eye spectral sensitivity $V(\lambda)$ as possible

Photometric calibration:

- Grey levels have to be converted into luminance values, taking the integration time into account

- The image uniformity has to be verified, by controlling that a uniform luminance distribution produces an image of equal grey values (CCD component sensitivity and lens effects)

Geometric calibration:

- The relation between the pixels co-ordinates of the final image and the corresponding outgoing light rays directions $\left(\theta_{2}, \phi_{2}\right)$ must be determined.

Additional possible sources of experimental errors, like the diffusion characteristics of the screen and the homogeneity of the incident light beam, must be checked to avoid losses of accuracy in the BTDF assessment.

${ }^{2}$ Image-Pro Plus ${ }^{\mathrm{TM}}$, v. 3.0.01.00, Media Cybernetics, L.P. 


\section{$\underline{\text { Spectral calibration }}$}

To spectrally calibrate the CCD camera, its sensitivity is determined experimentally, by a colour separation of light through a monochromator and a comparative analysis of the emerging ray by the camera and a calibrated luminance-meter; a least-square method is then applied to select the optimal optical filters to correct the spectral sensitivity and make it best fit $\mathrm{V}(\lambda)$ [12]: the function to minimise is given by (2); details on this procedure may be found in [13].

$$
\sum_{k=1}^{N}\left[\frac{1}{\left(V\left(\lambda_{k}\right)+0.01\right)} \cdot\left(V\left(\lambda_{k}\right)-0.92^{\left(N_{F}-\sum_{n=1}^{N_{F}} \frac{e_{n}}{e_{n 0}}\right)} \cdot \zeta \cdot S\left(\lambda_{k}\right) \cdot \prod_{n=1}^{N_{F}}\left(T_{n 0}\left(\lambda_{i}\right)\right)^{e_{n} / e_{n 0}}\right)^{2}\right]
$$

where

$\mathrm{N}$ : Number of measured points during spectral calibration [-]: $\mathrm{N}=34$

$\mathrm{N}_{\mathrm{F}}$ : Number of filters [-]: $\mathrm{N}_{\mathrm{F}}=3$

$S\left(\lambda_{\mathrm{k}}\right)$ : Relative spectral sensitivity of the camera [-]

$\mathrm{T}_{\mathrm{n} 0}$ : Transmission of filter $\mathrm{n}$ at thickness $\mathrm{e}_{\mathrm{n} 0}[-]$

$\mathrm{e}_{\mathrm{n}}$ : Thickness of filter $\mathrm{n}$ for optimal correction [mm]

$\mathrm{e}_{\mathrm{n} 0}$ : Thickness at which filter n's transmission $\mathrm{T}_{\mathrm{n} 0}$ has been measured [mm]

$\zeta$ : Scale factor used to normalise the experimental term in the least-square method [-]

The term 0.01 is added to minimise the relative error on points of low sensitivity; the 0.92 factor takes reflection effects into account, inducing a loss of transmitted light through optical filters. The scale factor $\zeta$ is expressed by equation (3):

$$
\zeta=\frac{1}{\max _{k=1}^{N}\left[0.92^{\left(N_{F}-\sum_{n=1}^{N_{F}} \frac{e_{n}}{e_{n 0}}\right)} \cdot S\left(\lambda_{k}\right) \cdot \prod_{n=1}^{N_{F}}\left(T_{n 0}\left(\lambda_{k}\right)\right) e^{e_{n} / e_{n 0}}\right]}
$$

\footnotetext{
${ }^{3}$ LMT LichtMeßTechnik GMBH Berlin
} 
The variables to determine are therefore the $e_{n}$ values. The choice of optical filters adapted to our purpose is based on photopic filters catalogues and on the $\mathrm{PhD}$ report [14]; the selected filters are Kopp n³384, Kopp n³307 and Schott BG39. The right thickness' are calculated with the Microsoft EXCEL ${ }^{\circledR}$ Solver, with the following limits: $\Sigma \mathrm{e}_{\mathrm{n}} \leq 8 \mathrm{~mm}$, in order to install them easily on the camera's objective, and $\mathrm{e}_{\mathrm{n}} \geq$ $0 \forall \mathrm{n}$.

The results are : $0.47 \mathrm{~mm}$ for the Kopp $n^{\circ} 3384$, 3.09mm for the Kopp $n^{\circ} 3307$, 1.20mm for the Schott BG39; the corresponding predicted transmissions and their combination are shown on Fig. 5.

Fig. 5. Mathematically predicted filter transmission curves, at calculated thickness'.

Once these filters are chosen and cut to the appropriate thickness', they are placed in front of the lens to achieve a quasi $V(\lambda)$ response. Fig. 6 shows the spectral response achieved by the CCD camera with and without correction filters; the human eye spectral sensitivity $V(\lambda)$ is also shown on the figure for comparison.

Fig. 6. Spectral calibration of the CCD camera.

\section{$\underline{\text { Photometric calibration }}$}

The relationship between picture grey levels and associated luminance values are determined for each used integration time ( $40 \mathrm{msec}$ to $2.56 \mathrm{sec}$ ), by simultaneous measurements of a white uniform screen illuminated by a 500 Watts halogen lamp placed at different distances, by the way of the CCD camera and a calibrated 
luminance-meter. The grey level to luminance conversion is illustrated by Fig. 7 for the different used integration intervals: non-linear response curves $(\gamma=0.45)$ are deliberately chosen to achieve a good resolution for low luminance levels.

Fig. 7. Photometric calibration for the different used integration times.

The image uniformity is checked to ensure that luminance measurements of different points on the image field are independent from their position on the latter. A stable luminous configuration, characterised by a constant luminance, is filmed through the CCD camera at different relative positions on the image field. The averaged values of different positions, obtained after calibration, are shown on Fig. 8: luminance relative differences of $3 \%$ are observed when approaching the triangle edges, with a relative darkening of $10 \%$ for the triangle apex. A software correction, explained in $\S$ 2.3, is applied to correct these small effects.

Fig. 8. Control of image uniformity. The given values are luminances $\left[\mathrm{cd} / \mathrm{m}^{2}\right]$, deduced from calibrated grey level images.

\section{$\underline{\text { Geometric calibration }}$}

To achieve the geometric calibration, the polar co-ordinates $\left(\theta_{2}, \phi_{2}\right)$ have to be converted into planar co-ordinates determining a point on the triangular screen; this geometrical transformation, illustrated by Fig. 9, is obtained through three dimensional trigonometric considerations and given by equations (4). The polar angle $\psi_{2}$ is comprised between $-30^{\circ}$ and $30^{\circ}$ and determines the azimuth value of a particular point for a given screen position; it is null along the central axis of the screen. 


$$
d=\frac{\sqrt{3} \cdot H}{2 \cdot\left(\sin \theta_{2} \cdot \cos \psi_{2}+\frac{\sqrt{3}}{2} \cos \theta_{2}\right)} \quad i=d \cdot \sin \theta_{2} \cdot \sin \psi_{2} \quad j=d \frac{\cos \theta_{2}}{\sin \Theta_{0}}
$$

where

d: Distance from sample centre to screen along direction $\left(\theta_{2}, \psi_{2}\right)$ (or $\left.\left(\theta_{2}, \phi_{2}\right)\right)[\mathrm{m}]$

H: Vertical distance from screen apex to base plane $(\mathrm{H}=1.15)[\mathrm{m}]$

$\Theta_{0}$ : Fixed angle between screen plane and main platform $\left(\Theta_{0}=\right.$ atan $\left.2 / \sqrt{3}\right)\left[{ }^{\circ}\right]$

(i, j): Cartesian screen co-ordinates [m]

Fig. 9. Relation between polar co-ordinates $\left(\theta_{2}, \psi_{2}\right)$ and associated cartesian screen co-ordinates (i,j).

Image distortions, due to possible imperfections of the camera lenses, are taken into account without adding a supplementary geometrical correction factor to equation (4).

For that purpose, a grid, composed of lines of different altitude angles $\theta_{2 n}$ and azimuth angles $\psi_{2 \mathrm{n}}$ is drawn on a screen and exposed to the camera. The screen has the same thickness and dimensions as the diffusing triangular panel installed in the photogoniometer: Fig. 10A shows the map screen, defining 901 different points at the lines intersections for angular resolutions of $2.5^{\circ}$ in both directions.

The same type of grid is used to define the angular resolution on the "transmission hemisphere” of the outgoing directions of light rays: angular resolutions of $10^{\circ}$ for altitude angle and $15^{\circ}$ for azimuth are shown as an example on Fig. 10B.

Fig. 10. A Support grid for geometric calibration. B Outgoing directions grid for $\left(\Delta \theta_{2}, \Delta \phi_{2}\right)=\left(10^{\circ}, 15^{\circ}\right)$.

\section{$\underline{\text { Additional corrections }}$}

Other possible sources of error are checked by complementary measures. 
The diffusion characteristics of the triangular screen, together with the uniformity of the reflection coefficient, have been measured by the way of two experimental setups.

A Minolta CR-200b surface chromameter was used to measure the reflection coefficient of different points over the whole screen, showing a mean value for $\rho$ of 0.743 , with relative fluctuations lower than $0.7 \%$. Luminance values, measured at different emerging angular directions from the triangular panel, were used to assess the diffusion characteristics of the LMT white paint: a Lambertian diffusion was ideally expected to be observed.

Fig. 11 shows the measured luminance distribution, which fits to a Lambertian model curve within a $10 \%$ relative range; this minor imperfection in diffusion will be corrected as detailed in $\S 2.3$. Grazing incidence leads, however, to poorer results, showing a significant component of specular reflection: this does not have serious impact on the photogoniometer's performances, incidence angles on the triangular projection panel being lower than $50^{\circ}$ in any case (by the way of geometrical position of the sample).

Fig. 11. Measured luminance distribution after reflection on LMT photometer paint, for different incident directions: A Incidence $10^{\circ}$. B Incidence $45^{\circ}$. The theoretical values are deduced from the lambertian model (ideal diffusion).

The light beam uniformity over the sample area has been checked, showing fluctuations lower than $2 \%$ in relative terms. Another analysis concerns the collimation of the incident beam, a significant divergence causing an error in the area factor (see $\S 2.3$ ). An experimental procedure was therefore carried out to estimate the possible angular spread of the beam by comparing the measured and the 
theoretical diameter of a zone defined by the light beam on the triangular screen, when passing through the aperture of the photogoniometer (sample position). The projected diameter is determined experimentally by applying a line contour on the image and integrating the corresponding values (see Fig. 12) ; the theoretical profile is deduced assuming a perfectly collimated beam (parallel rays), showing no scattering nor divergence. By comparing both projections, the incident light divergence can be calculated : it appears to be close to $0.35^{\circ}$. However, the real sunlight divergence being of $0.25^{\circ}$, we can admit this situation as an acceptable reference and choose to correct only the source beam's excess in divergence with regard to this reference. In consequence, a 1.04 correction factor is found and shall be applied to the diameter of the diaphragm, which corresponds to a correction of $8 \%$ in relative terms on the area $\left(\mathrm{A}=\pi \mathrm{D}^{2} / 4\right)$.

Fig. 12. Illuminated zones' line profile compared to a theoretical step for an incidence normal to the screen surface $\left(\theta_{1}=\Theta_{0}\right)$.

\subsection{Image and data processing}

The driving software of the photogoniometer was written in VISUAL BASIC ${ }^{\circledR}$ to achieve a fully automated sample characterisation. The default incident directions set comprise 145 different angular directions, matching the subdivision of the sky hemisphere for luminance measurements defined by Tregenza for the International Daylighting Measurement Program [15] (Fig. 13); any other angular distribution can be chosen if desired. Only 2 to 4 minutes are necessary to acquire a complete set of BTDF data for one incident direction versus hours for classical photogoniometers. 
Fig. 13. Driving software interface for sample characterisations. There are mainly three ways of exploring the incident hemisphere (Tregenza distribution, steps in altitude along C planes, free or regular angular steps), combined to a graphical illustration of the characterisation's evolution.

The aim of the image processing is to improve the accuracy of luminance measurements and avoid over-exposure and/or under-exposure of some parts of the digital images, in presence of high luminance dynamics.

An automated selection of appropriate integration intervals, followed by an addition of the concerned image parts, is achieved by the driving software to match the luminance dynamic [16]. For each six screen positions, picture snapshots are taken at different integration intervals, the largest one being just small enough to avoid overexposure and the lowest one being just large enough to avoid under-exposure. All these images are based on appropriate pixels calibrations from grey level to luminance, converted into 32 bits (floating point) images and divided by the illuminance simultaneously measured on the sample plane $E_{1}\left(\theta_{1}\right)$ : the value of each pixel, in consequence, is equal to the ratio of the corresponding "screen luminance" $\mathrm{L}_{\text {screen}}$, sensed by the CCD camera, to the sample plane illuminances $\mathrm{E}_{1}\left(\theta_{1}\right)$. The images, placed in the order of the brightest to the darkest, are then added to form a completely calibrated 32 bits image (all the over-exposed or already treated pixels being set to null during this process).

As the camera and the sample remain within the same relative positions and directions with regard to the screen, the effects of image non-uniformity and non- 
perfect diffusion remain constant on the final treated image: both effects can therefore be corrected through the same procedure.

Screen snapshots are taken for two kinds of situations:

- $\quad$ with a lambertian diffuser, showing a rotational symmetry, placed on the aperture (Fig. 14A)

- with no sample on the aperture, leaving the light beam reach the triangular panel without any perturbation (Fig. 14B).

Fig. 14. A Lambertian diffuser. Points of equal altitude $\theta_{2}$ are expected to be of equal luminance, once their distance and light tilting effects have been compensated. B Superposition of images obtained without any sample (hole). The centre pixels inside each illuminated zone are expected to have values deduced from relation (5).

The first configuration is used to determine groups of pixels of expected equal luminance, taking distance and light tilting effects into account (see equation (6) below): these groups of pixels are characterised by equal $\theta_{2}$ values, as shown on Fig. 14A. The values of the pixels belonging to the central screen axis are considered as a reference for each group; correction factors are thus determined by comparison for the other pixels inside the groups, and then interpolated in-between.

The second configuration can be used to assess a theoretically expected screen luminance given by equation (5), obtained under the consideration of a perfectly diffusing surface (see Fig. 15):

$E_{P}=\frac{I_{0} \cos \left(\theta_{1}\right)}{h^{2}} ; \quad E_{P^{\prime}}=\frac{I_{0} \cos \alpha}{(h+d)^{2}}=\frac{\pi L_{P^{\prime}}}{\rho} \quad ; \quad \frac{L_{P^{\prime}}}{E_{P}}=\frac{\rho E_{P^{\prime}}}{\pi E_{P}}=\frac{\rho h^{2} \cos \alpha}{\pi(h+d)^{2} \cos \theta_{1}}$

where

$\mathrm{I}_{0}$ : Light source intensity [cd] 
h: Distance from sample to light source [m]

$E_{P}$ : Illuminance of the sample plane, at point $\mathrm{P}[\mathrm{lx}]$

$\mathrm{E}_{\mathrm{P}}$ : Illuminance of the triangular screen, at point $\mathrm{P}^{\prime}[\mathrm{lx}]$

$\mathrm{L}_{\mathrm{P}}$ : Luminance of reflected light flux at point $\mathrm{P}^{\prime}\left[\mathrm{cd}^{\cdot} \mathrm{m}^{-2}\right]$

$\rho$ : Reflection factor of triangular screen [-]

$\alpha$ : Angle between normal to screen and direction $\left(\theta_{2}, \psi_{2}\right)\left[{ }^{\circ}\right]$

Fig. 15. Geometric characteristics of an incident beam reaching the screen without any sample.

Practically, the calibrated pixel values on the images are divided by the illuminance simultaneously measured on the sample plane $E_{P}=E_{1}\left(\theta_{1}\right)$ in order to compensate any light source fluctuation effects; the images are thus composed of pixels presenting $\mathrm{L}_{\mathrm{P}} / \mathrm{E}_{\mathrm{P}}$ ratios as values. The theoretical values expected along the central screen axis may be compared to the ones obtained on the calibrated "hole" images (Fig. 14B); a correction curve for the central axis can be deduced: it is represented in Fig. 16.

Fig. 16. Correction factor evolution along central screen axis.

The correction is then extended to all other pixels, by multiplying the results of both configurations : the factors obtained (0.88 through 1.13) are represented in Fig. 17 by grey levels.

During its processing, each final image is thus multiplied by this correction figure to compensate non-uniformity and imperfect diffusion of the triangular screen. 
Fig. 17. Correction figure for image non-uniformity and imperfect diffusion of triangular screen: grey levels are to be associated with correction factor values: 0.88 (black) to 1.13 (white) ; the image region outside the measured area (screen) is arbitrarily fixed to 1.

The corrected pixel values of the end images are finally averaged to meet a certain angular discretisation grid, for example each $10^{\circ}$ in altitude and $15^{\circ}$ in azimuth (i.e. $\Delta \theta_{2}=10^{\circ} ; \Delta \phi_{2}=15^{\circ}$ ) like illustrated in Fig. 10B ; the values obtained are converted into BTDF values (coming out from the sample centre) using the analytical equation given by (6). This equation corrects the distance and the light tilting effects; it has been obtained by considering the triangular screen as a perfect diffusing surface:

$$
\operatorname{BTDF}\left(\theta_{1}, \phi_{1}, \theta_{2}, \phi_{2}\right)\left[\frac{c d}{m^{2} l x}\right]=\frac{\pi}{\rho} \cdot \frac{d^{2}\left(\theta_{2}, \psi_{2}\right)}{A \cdot \cos \theta_{2} \cdot \cos \alpha} \cdot \frac{L_{\text {screen }}\left(\theta_{1}, \phi_{1}, \theta_{2}, \phi_{2}\right)}{E_{1}\left(\theta_{1}\right)}
$$

where $\mathrm{A}$ is the illuminated area of the sample $\left[\mathrm{m}^{2}\right]$.

\section{Experimental results}

The BTDF values can be saved in an ASCII file, containing the sample characteristics (name, manufacturer, thickness, etc.), the measurement type (incident direction, output angular resolution, etc.) and the hemispherical light transmittance $\tau\left(\theta_{1}, \phi_{1}\right)$, calculated by integration of the BTDF values through the approximation given by equation (7):

$$
\tau\left(\theta_{1}, \phi_{1}\right) \approx \Delta \theta_{2} \cdot \Delta \phi_{2} \cdot \sum_{n=1}^{N_{\text {out }}} B T D F_{n}\left(\theta_{1}, \phi_{1}, \theta_{2 i}, \phi_{2 i}\right) \cdot \cos \theta_{2 n} \cdot \sin \theta_{2 n}
$$

where $\mathrm{N}_{\text {out }}$ is the number of angular directions, discretising the outgoing hemisphere. 
It must be noted that the calculation of $\tau$ appears to be heavy and tedious in case of irregular output angular resolutions, which are often used for conventional photogoniometric measurements (mobile photometer) [8]. Thank to digital imaging techniques, this problem disappears, the continuity of the information leading to an exclusion of any risk of missing an important feature in transmission.

The BTDF values are given with the outgoing associated angular directions. In order to avoid any confusion between files providing BTDFs and BRDFs (bi-directional reflection distribution functions), the altitude angle $\theta_{2}$, defined as the supplementary angle of $\theta_{2}$ (i.e. as the angle between the normal to the sample on the incident interface and the emerging (transmitted) light flux direction: $\theta_{2}^{\prime}=180-\theta_{2}$ ), is used to give the outgoing direction in these final data files; it is therefore comprised between $90^{\circ}$ and $180^{\circ}$.

As a result of image processing, the achieved data represent average values of the BTDFs measured in the output hemisphere regions limited by $\left(\phi_{2}-1 / 2 \Delta \phi_{2} ; \phi_{2}+\right.$ $\left.1 / 2 \Delta \phi_{2}\right)$ in azimuth and by $\left(\theta_{2}-1 / 2 \Delta \theta_{2} ; \theta_{2}+1 / 2 \Delta \theta_{2}\right)$ in altitude for each outgoing direction.

\subsection{Graphical data processing}

The six calibrated images created for the six screen positions are superposed to build up an image showing the directional transmission, represented in polar co-ordinates (Fig. 18A and 19A). The final built up of the image, on which non-homogeneity and imperfect screen diffusion have already been compensated (Fig. 17), includes the 
different corrections for distance and light tilting effects, accounted through equation (6).

The set of BTDF numerical data is very large and cannot by itself give a synthetic view of the sample's transmission features. A graphical processing is therefore necessary to provide a reasonable appreciation of the light transmission behaviour. The data are treated for this purpose by MATLAB ${ }^{\circledR 4}$ to create three dimensional graphical representations of BTDFs; examples are shown on Fig. 18B, 18C, and 18D of different visualisation possibilities of the transmission feature of a 3M prismatic film under an incidence of $\left(40^{\circ}, 0^{\circ}\right)$, and on Fig. 19B, 19C, and 19C for a pleated tissue blind manufactured by Baumann-Hüppe AG under normal incidence. Fig. 18B and 19B represent BTDF values projected on a virtual hemisphere (the colour scale is proportional to the BTDF values); Fig. 18C and 19C show the photometric solid (same colour scale), built up in spherical co-ordinates. For both representations, the viewpoint can be freely chosen by mouse clicking. Fig. 18D and 19D give section views along $\mathrm{C}$ planes (perpendicular to sample plane) of the built photometric solid, which yield to a clear quantitative analysis of the BTDF behaviour.

On these figures, one can easily observe a splitting of the incident beam into two non-trivial outgoing directions for the prismatic film, and a privileged plane for transmission induced by the pleats for the tissue blind. This clear-sighted visualisation of the material's transmission features allow a good understanding of its photometric performances when used as a fenestration material.

${ }^{4}$ MatLAB(, v. 5.3.0.10183, The MathWorks, Inc. 
Fig. 18. Prismatic SOLF film (3M) : light incidence on prismatic side; $\left(\theta_{1}, \phi_{1}\right)=\left(40^{\circ}, 0^{\circ}\right) ; \varnothing=10 \mathrm{~cm} ;\left(\Delta \theta_{2}\right.$, $\left.\Delta \phi_{2}\right)=\left(5^{\circ}, 5^{\circ}\right)$. A Calibrated image recomposed from sixths. B Hemispherical projection. C Photometric solid. D Section views along C planes.

Fig. 19. Pleated tissue blind (Baumann-Hüppe AG) : $\left(\theta_{1}, \phi_{1}\right)=\left(0^{\circ}, 0^{\circ}\right) ; \varnothing=17 \mathrm{~cm} ;\left(\Delta \theta_{2}, \Delta \phi_{2}\right)=\left(10^{\circ}, 15^{\circ}\right) . \quad \mathbf{A}$ Calibrated image recomposed from sixths. B Hemispherical projection. C Photometric solid. D Section views along C planes.

\subsection{Experimental validation}

As most bi-directional photogoniometers are generally under testing stages, there is no extended BTDF data set accurate enough to be taken as a reference for validation purpose. The possibilities to validate the equipment are therefore of different kind: a) comparison with analytically expressed BTDF values, for well-known situations (an open hole or a lambertian sample), b) comparison of integrated BTDF values and hemispherical transmission measured with an Ulbricht sphere [6], c) comparison of BTDF values of the same sample measured with different photogoniometers [7] [8] [9].

The first two validation ways were applied on our earliest results, as illustrated on Fig. 20, showing discrepancies lower than 10\%. A comparison of BTDFs will be carried out as soon as a larger data set will be available.

Fig. 20. Integrated light transmittance of prismatic SOLF film (3M), with light incidence on prismatic side. The Integrating Spheres results are based on an average value obtained from measurements of the same samples in different laboratories, with a standard deviation given by the error bars. 


\section{Conclusion}

This novel bi-directional photogoniometer has been tested successfully and the advanced imaging techniques have proven their usefulness to speed up the data acquisition process and improve the resolution in luminance. The difficulty lies in the high quality calibration requested for the CCD camera, in order to convert it into a reliable calibrated multiple points luminance-meter.

The image processing allows to get rid of any over or under-exposure effect, with a guarantee of equal and accurate resolution over the whole luminance dynamic. The possibility to scan the hemisphere of outgoing rays in a continuous way provides not only appropriate global representations, but gives the insurance to take every peak or trough - in transmission into account, as narrow it could be. Moreover, the time needed to perform a complete measurement, considerably reduced by the use of digital imaging (2 to 4 minutes per incident direction), does not depend on the output angular resolution required, contrarily to a point per point analysis inherent to conventional photogoniometers, fitted with movable photometers. Moreover, the graphical representations of the BTDF values in spherical co-ordinates provide a synthetic and intuitive approach of the light transmission distribution, which is appropriate for the judicious choice and improvement of glazing materials. Effectively, a thorough knowledge of the fenestration elements' transmission features will allow an optimisation of these materials as far as their industrial conception is concerned, and an easier selection by the architect at the project's level. Several daylighting activities will certainly take benefit from accurate and objective characterisation of fenestration components, which can lead to a better use of these 
elements in building construction and allow progress in research and industrial applications. Further developments, combined with the creation of a BTDF data set for a statistically valid number of fenestration materials, could allow classifying them into certain typical transmission figures and providing a catalogue of market products to be used by architects and industrials.

\section{Acknowledgements}

The bi-directional photogoniometer has been set up thank to the financial and human support of the Swiss Federal Institute of Technology (EPFL) and the Commission for Technology and Innovation (CTI). The authors wish to thank P. Loesch for his work concerning the mechanical aspects of this device, and D. Glauser for his robotic concept. Thanks are also due to H.-P. Baumann, former director of Baumann Storen AG, for his final use of the photogoniometer.

\section{Nomenclature}

$\left(\theta_{1}, \phi_{1}\right)$ : Polar co-ordinates of incoming light flux $\left[^{\circ}\right]$

$\left(\theta_{2}, \phi_{2}\right)$ : Polar co-ordinates of emerging (transmitted) light flux $\left[^{\circ}\right]$

$\Delta \theta_{2}, \Delta \phi_{2}$ : Output angular resolution $\left[^{\circ}\right]$

$\mathrm{L}_{1}\left(\theta_{1}, \phi_{1}\right)$ : Luminance of element of incoming light flux [cd m$\left.{ }^{-2}\right]$

$\mathrm{L}_{2}\left(\theta_{1}, \phi_{1}, \theta_{2}, \phi_{2}\right)$ : Luminance of emerging (transmitted) element of light flux [cd m$\left.{ }^{-2}\right]$

$\mathrm{d} \omega_{1}$ : Solid angle subtended by incoming light flux [sr]

$\mathrm{d} \omega_{2}$ : Solid angle subtended by emerging (transmitted) light flux [sr]

$\mathrm{E}_{1}\left(\theta_{1}\right)$ : Illuminance on sample plane, due to incident light flux [lx]

$\mathrm{N}$ : Number of measured points during spectral calibration [-]: $\mathrm{N}=34$

$\mathrm{N}_{\mathrm{F}}$ : Number of filters [-]: $\mathrm{N}_{\mathrm{F}}=3$ 
$S(\lambda)$ : Relative spectral sensitivity of the camera [-]

$\mathrm{T}_{\mathrm{n} 0}$ : Transmission of filter $\mathrm{n}$ at thickness $\mathrm{e}_{\mathrm{n} 0}[-]$

$\mathrm{e}_{\mathrm{n}}$ : Thickness of filter $\mathrm{n}$ for optimal correction [mm]

$\mathrm{e}_{\mathrm{n} 0}$ : Thickness at which filter n's transmission $\mathrm{T}_{\mathrm{n} 0}$ has been measured [mm]

$\zeta$ : Scale factor used to normalise the experimental term in the least-square method [-]

$\psi_{2}$ : Polar angle determining the azimuth value for a given screen position $\left[{ }^{\circ}\right]$

d: Distance from sample centre to screen along direction $\left(\theta_{2}, \psi_{2}\right)$ (or $\left.\left(\theta_{2}, \phi_{2}\right)\right)[\mathrm{m}]$

$\mathrm{H}$ : Vertical distance from screen apex to base plane $(\mathrm{H}=1.15)[\mathrm{m}]$

$\Theta_{0}$ : Fixed angle between screen plane and main platform $\left(\Theta_{0}=\right.$ atan $\left.2 / \sqrt{3}\right)\left[{ }^{\circ}\right]$

(i, j): Cartesian screen co-ordinates [m]

$\rho:$ Reflection factor of triangular screen [-]

$\alpha$ : Angle between normal to screen and direction $\left(\theta_{2}, \psi_{2}\right)\left[^{\circ}\right]$

A: Illuminated area of sample $\left[\mathrm{m}^{2}\right]$

$\tau\left(\theta_{1}, \phi_{1}\right)$ : Hemispherical light transmittance of sample under incident direction $\left(\theta_{1}\right.$,

$\left.\phi_{1}\right)[-]$

$\mathrm{N}_{\text {out }}$ : Number of angular directions, discretising the outgoing hemisphere [-]

\section{References}

[1] F. Moore, Concepts and practice of architectural daylighting, Van Nostrand Reinhold Company, New York, 1985, 290 pp.

[2] Swiss Federal Office of Conjonctural Problems, Appropriate daylight, EDMZ, Bern, 1995, 80 pp.

[3] J.-L. Scartezzini, B. Paule et al., Office Lighting, EDMZ, Bern, 1994, 100 pp. 
[4] J.-L. Scartezzini, R. Compagnon, G. Ward, B. Paule, Computer daylighting simulation tools, Scientific Report, University of Geneva / EPFL, Lausanne, 1994, 142 pp.

[5] Commission Internationale de l'Eclairage, Radiometric and photometric characteristics of materials and their measurement, $2^{\text {nd }}$ Edition, CIE 38 (TC-2.3), Paris, 1977, 214 pp.

[6] S. Aydinli (ed.), Report on physical quantities to be measured in laboratory facilities, IEASHC Task21, Berlin, TUB, 1999, 50 pp.

[7] K. Papamichael, J. Klems, S. Selkowitz, Determination and Application of Bidirectional Solar-Optical Properties of Fenestration Systems, LBL-25124, CA, Lawrence Berkeley Laboratory, 1988, 8 pp.

[8] P. Apian-Bennewitz, Designing an apparatus for measuring bidirectional reflection/ transmission, SPIE 0-8194-1564-2 (2255), Freiburg, Fraunhofer Institute for Solar Energy Systems, 1994, 10 pp.

[9] L. Bakker, D. van Dijck, Measuring and processing optical transmission distribution functions of TI-materials, Private Communication, Delft, TNO, 1995, 4 pp.

[10] J.L.J. Rosenfeld, The new Cardiff goniometer to measure the detailed optical properties of advanced glazing units, Private Communication, Cardiff, University of Wales, 1997, 5 pp.

[11] J.-L. Scartezzini, R. Compagnon, C. Roecker, L. Michel, Bidirectional photogoniometer for advanced glazing materials based on digital imaging techniques, Lighting Research and Technology (29), London, CIBSE, 1997, 9 pp. 
[12]H. Wright, C.L. Sanders, D. Gignac, Design of Glass Filter Combinations for Photometers, Applied Optics (8), 1969, 7 pp.

[13]M. Andersen, Dispositif de mesure de la luminance du ciel basé sur des techniques d’imagerie numérique: développement et validation expérimentale, Diploma Report, Lausanne, EPFL, 1998, 97 pp.

[14]V. Berrutto, Métrologie de la qualité visuelle des ambiances lumineuses. Application à l'éclairage des bureaux, PhD Report, Université de Savoie, Chambéry, 1996, 209 pp.

[15] P.R. Tregenza, Subdivision of the sky hemisphere for luminance measurements, Lighting Research and Technology (19), London, CIBSE, 1987, 2 pp.

[16]L. Michel, Méthode expérimentale d’évaluation des performances lumineuses de bâtiments, PhD Report, Lausanne, EPFL, 1999, 209 pp. 


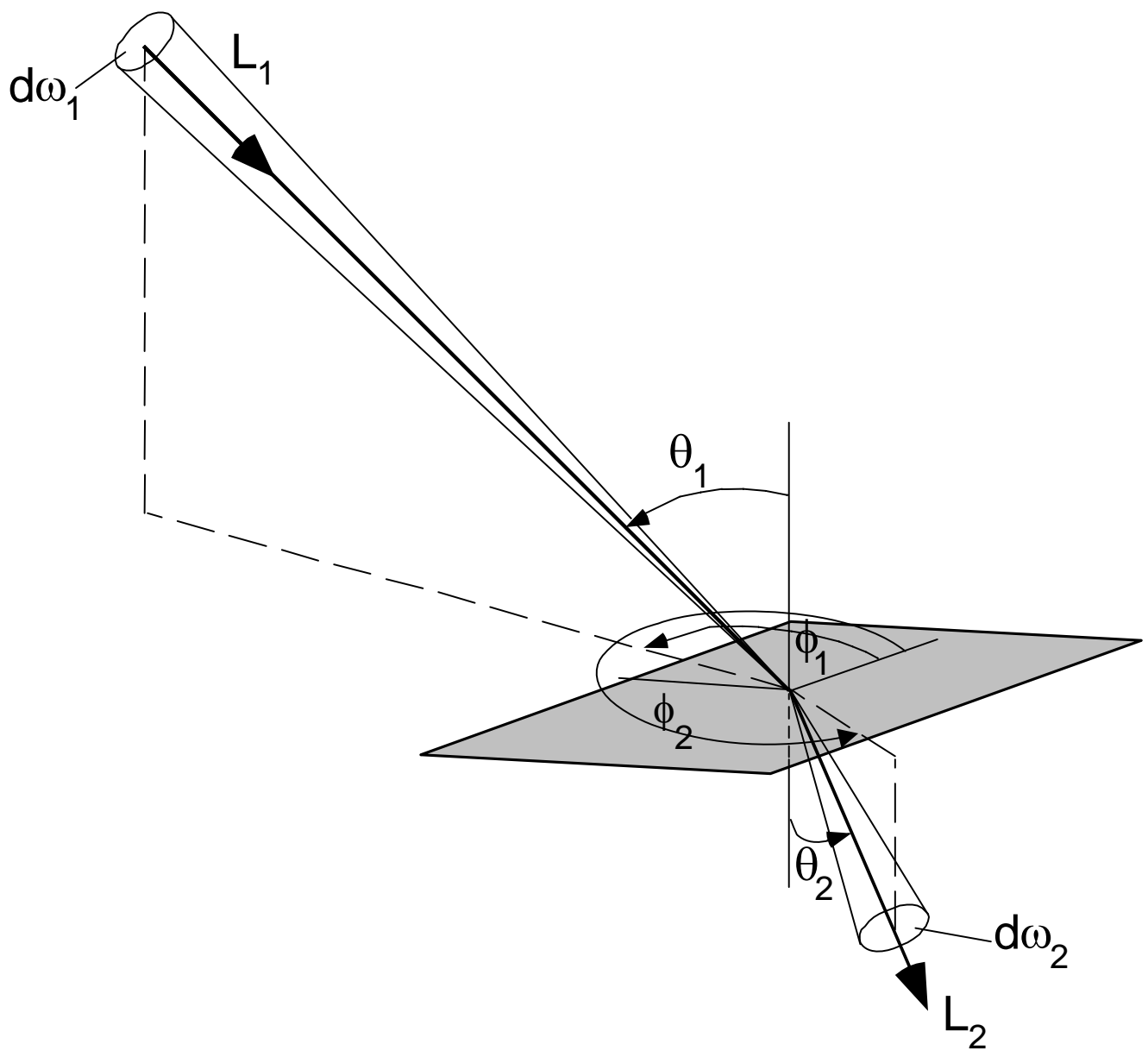

Fig. 2. Photometric and geometric quantities used for BTDF definition. 


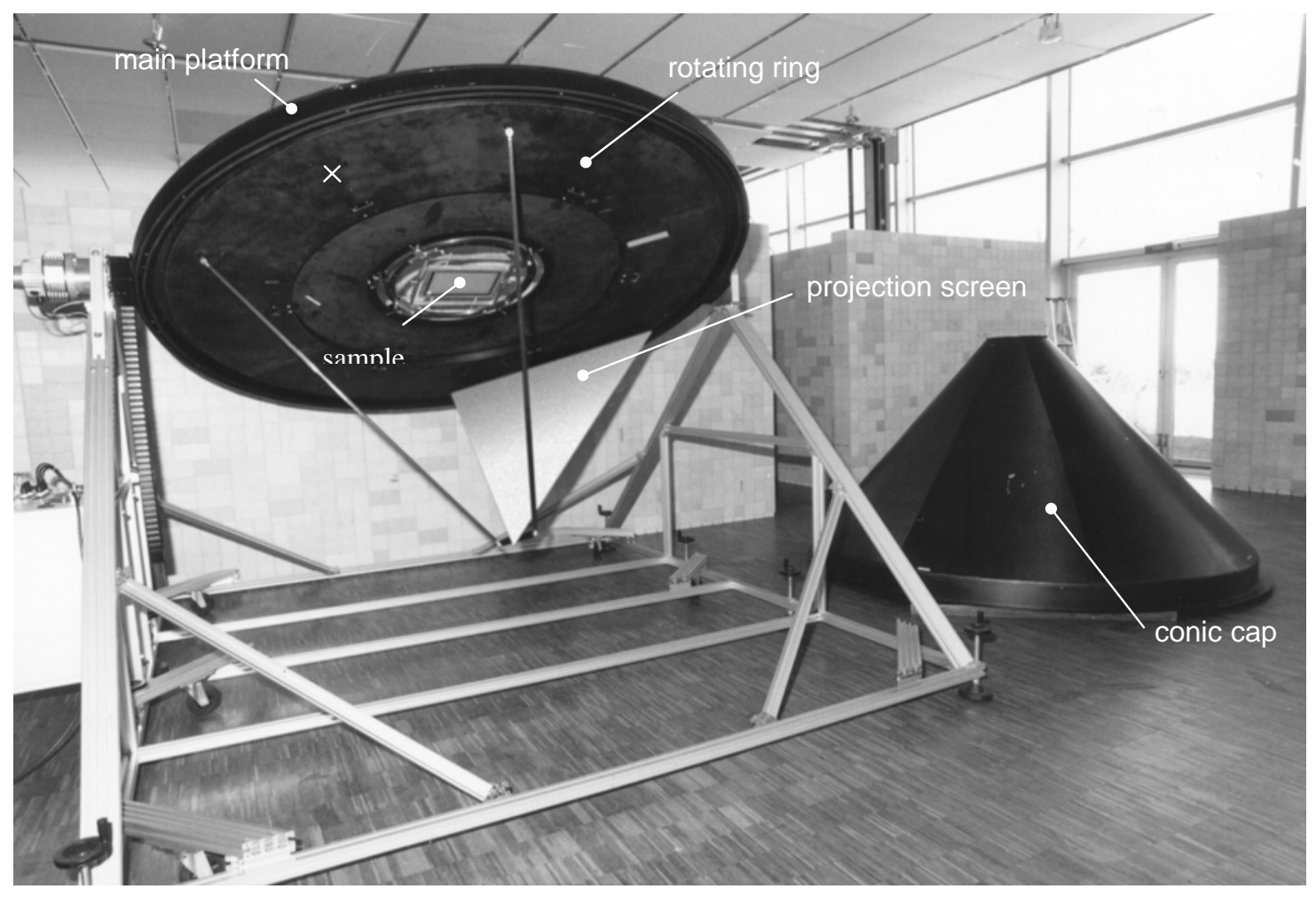

Fig. 2. Bi-directional photogoniometer developed at LESO-PB/EPFL. The CCD camera (absent on the picture) is fixed on the rotating ring at the cross mark. 


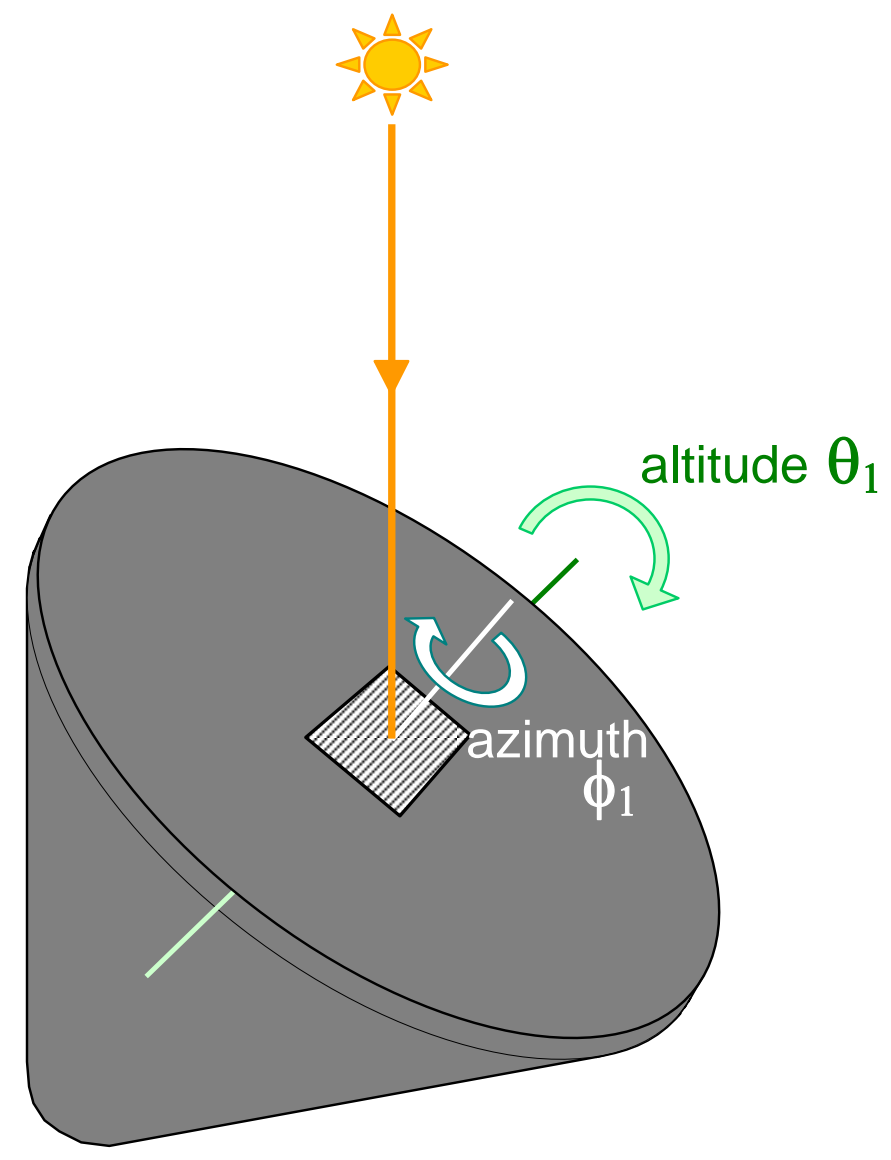

Fig. 3. Rotation of main platform and sample holder. 


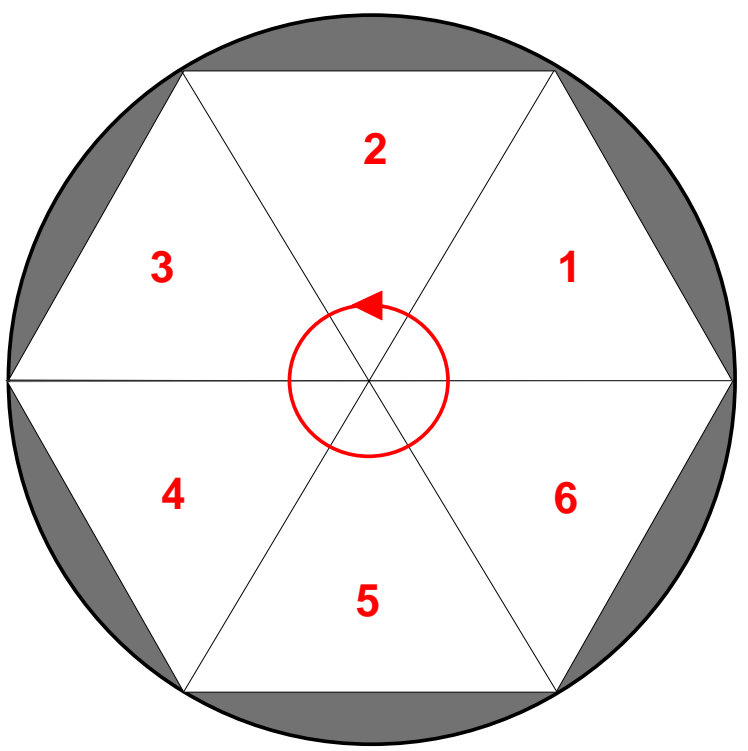

Fig. 4. Rotation of lambertian screen (orthogonal projection on the sample plane). 


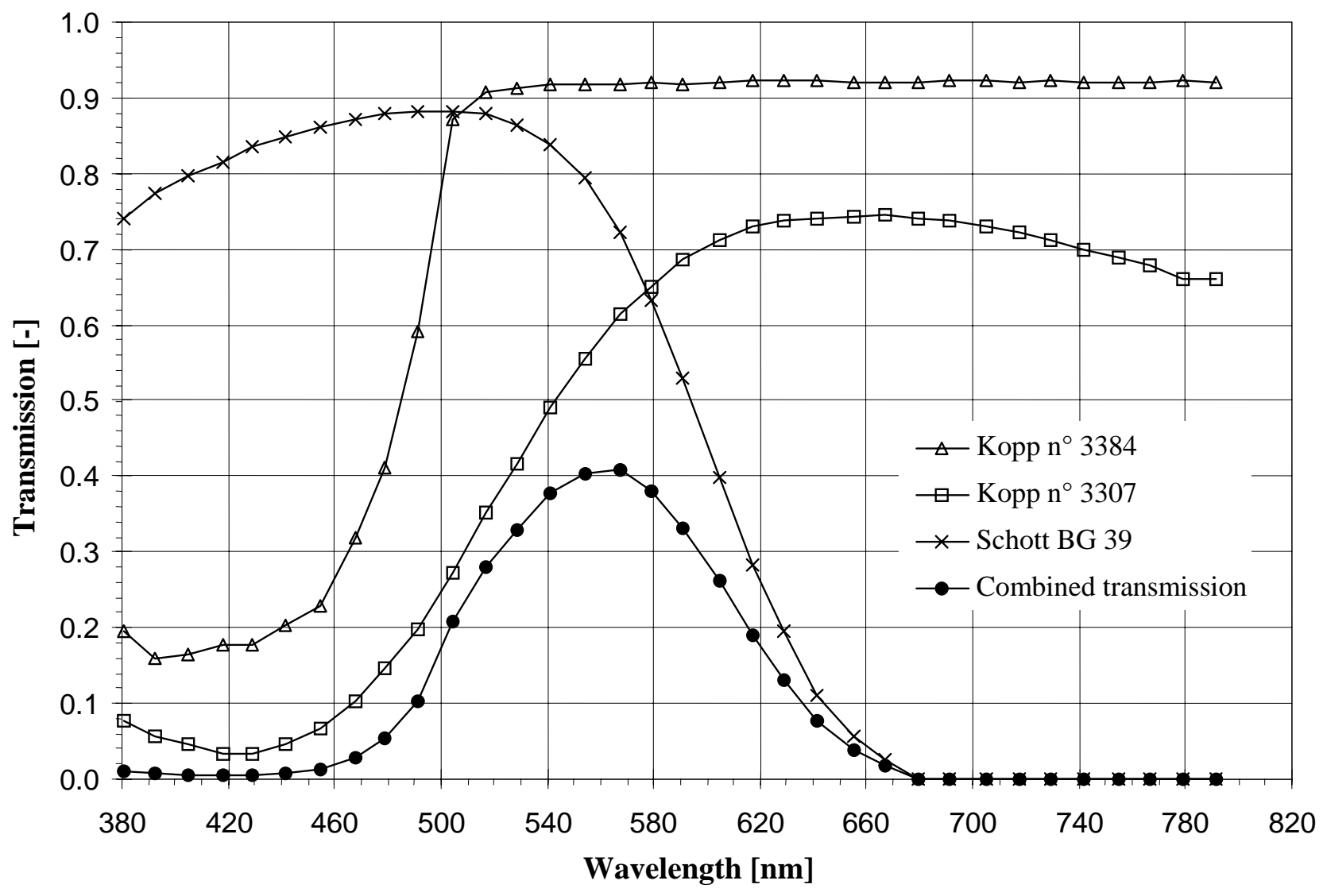

Fig. 5. Mathematically predicted filter transmission curves, at calculated thickness'. 


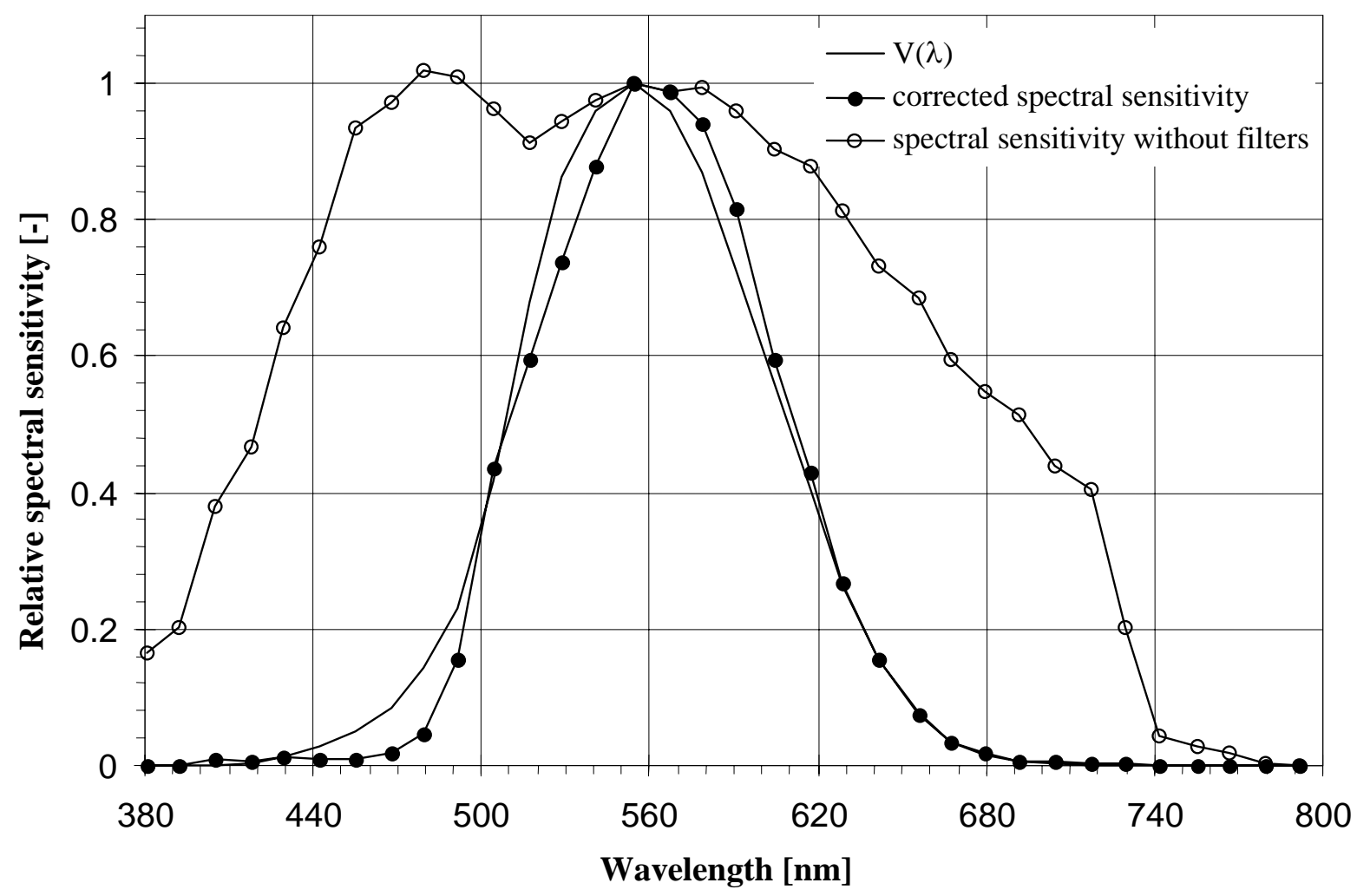

Fig. 6. Spectral calibration of the CCD camera. 


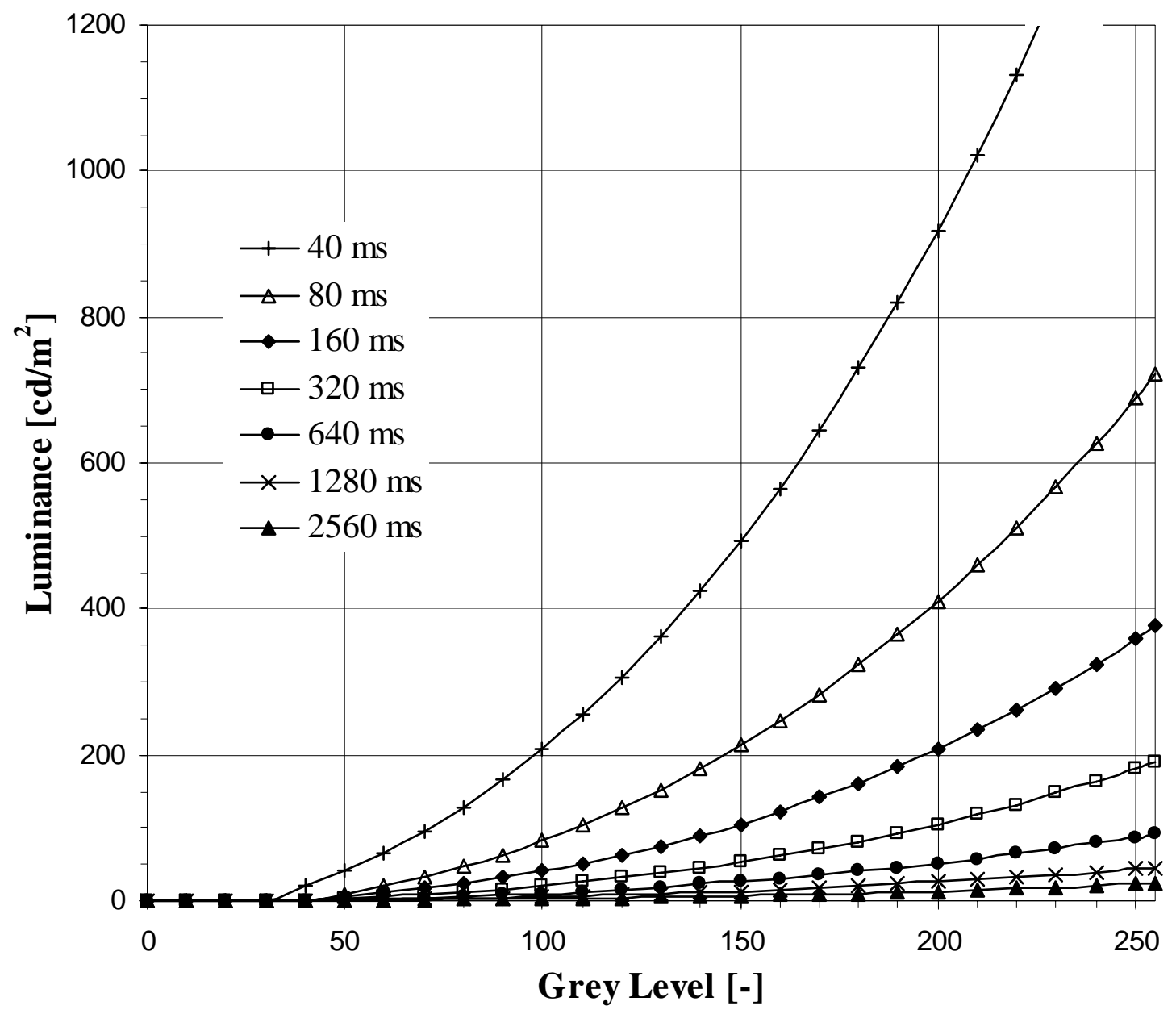

Fig. 7. Photometric calibration for the different used integration times. 


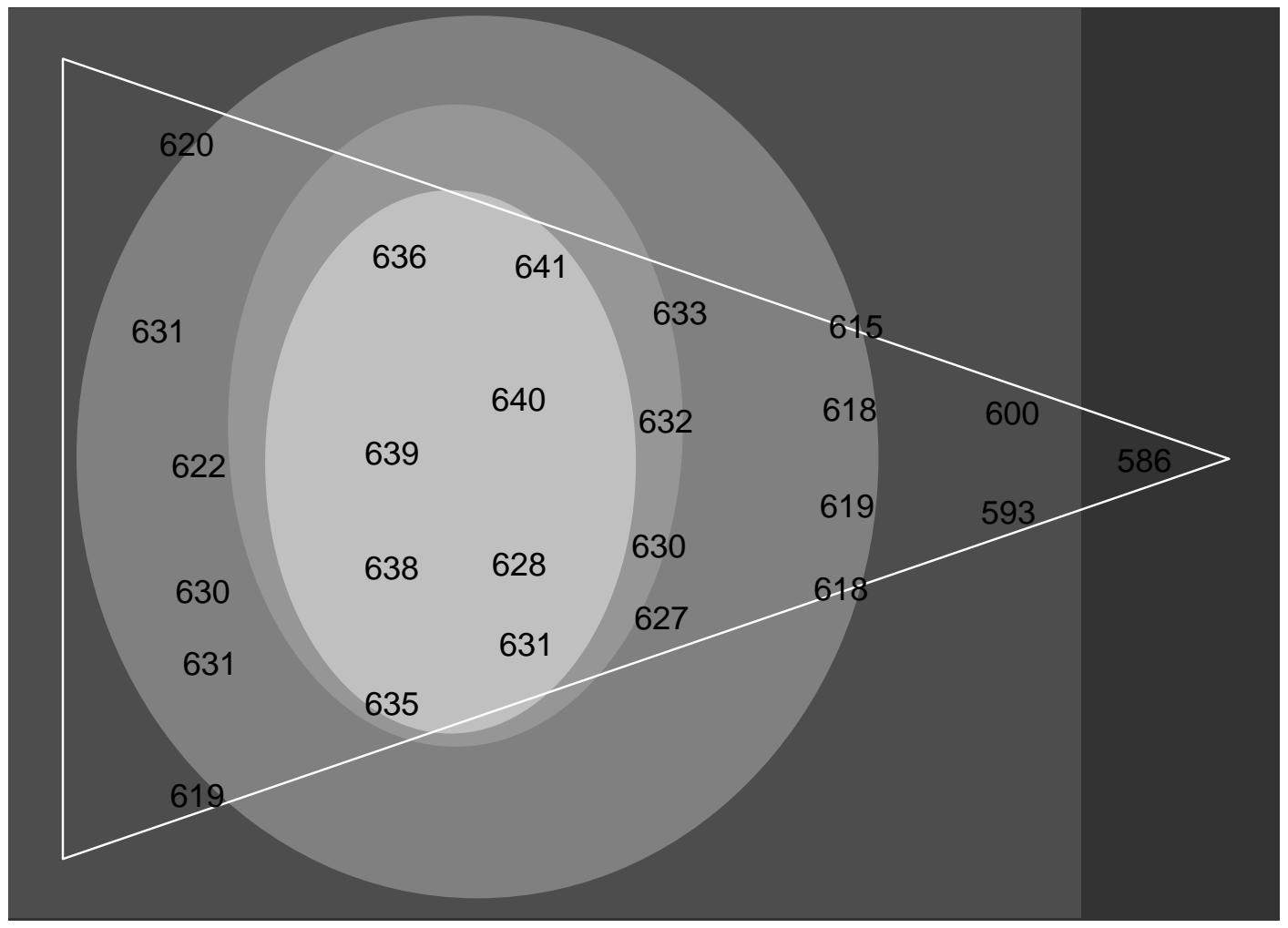

Fig. 8. Control of image uniformity. The given values are luminances $\left[\mathrm{cd} / \mathrm{m}^{2}\right]$, deduced from calibrated grey level images. 


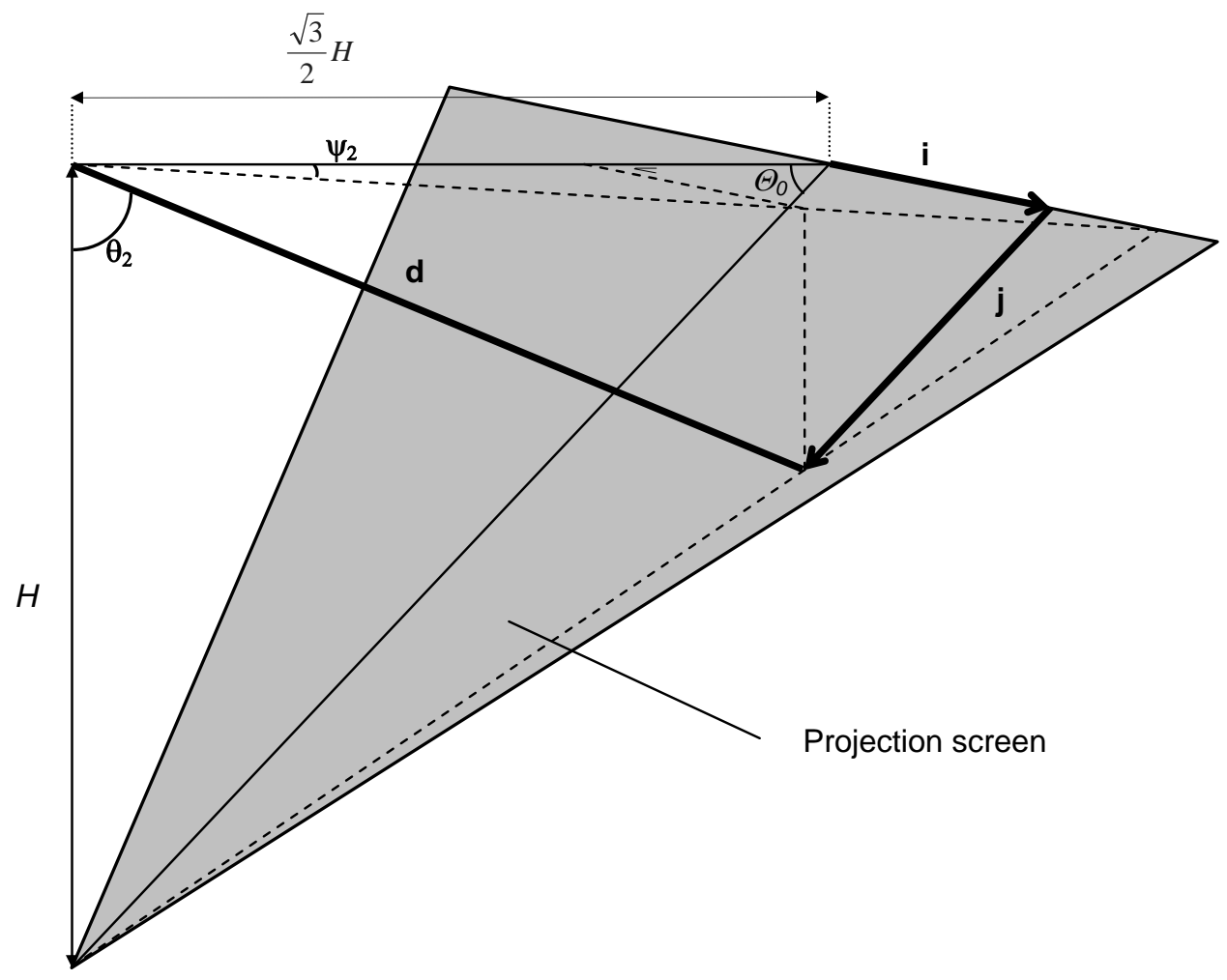

Fig. 9. Relation between polar co-ordinates $\left(\theta_{2}, \psi_{2}\right)$ and associated cartesian screen co-ordinates (i,j). 

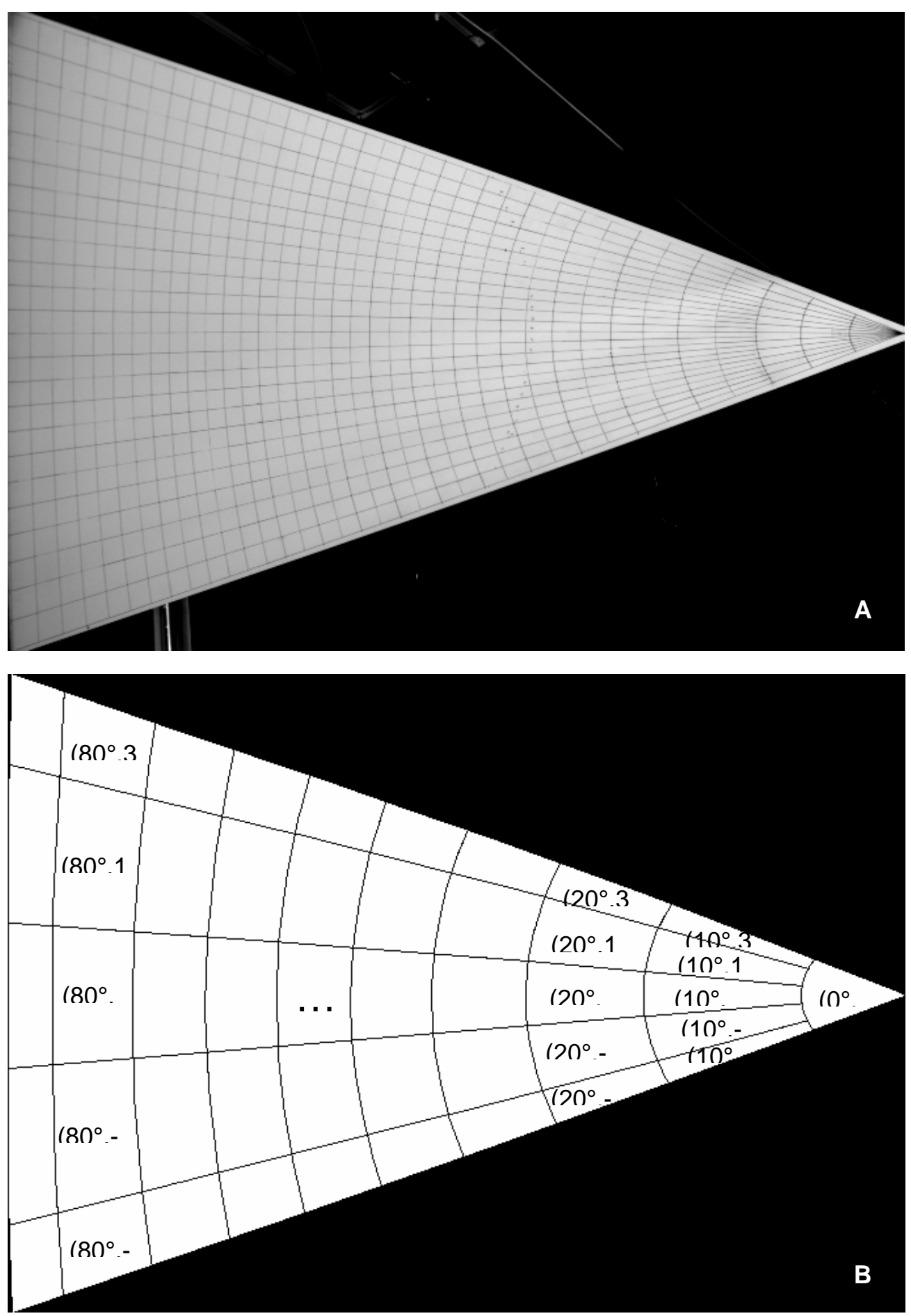

Fig. 10. A Support grid for geometric calibration. B $\left(\Delta \theta_{2}, \Delta \phi_{2}\right)=\left(10^{\circ}, 15^{\circ}\right)$. 

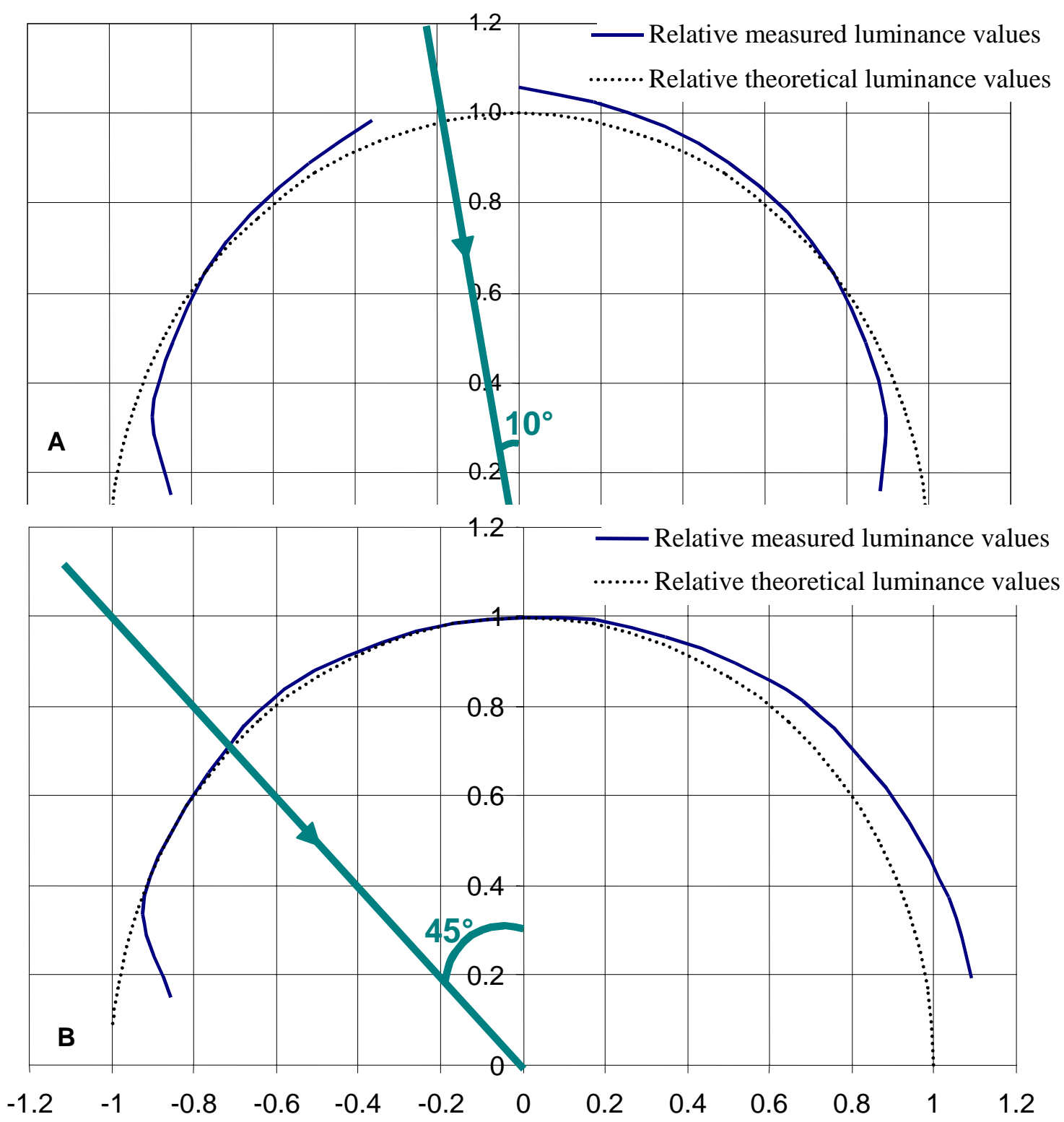

Fig. 11. Measured luminance distribution after reflection on LMT photometer paint, for different incident directions: A Incidence $10^{\circ}$. B Incidence $45^{\circ}$. The theoretical values are deduced from the lambertian model (ideal diffusion). 


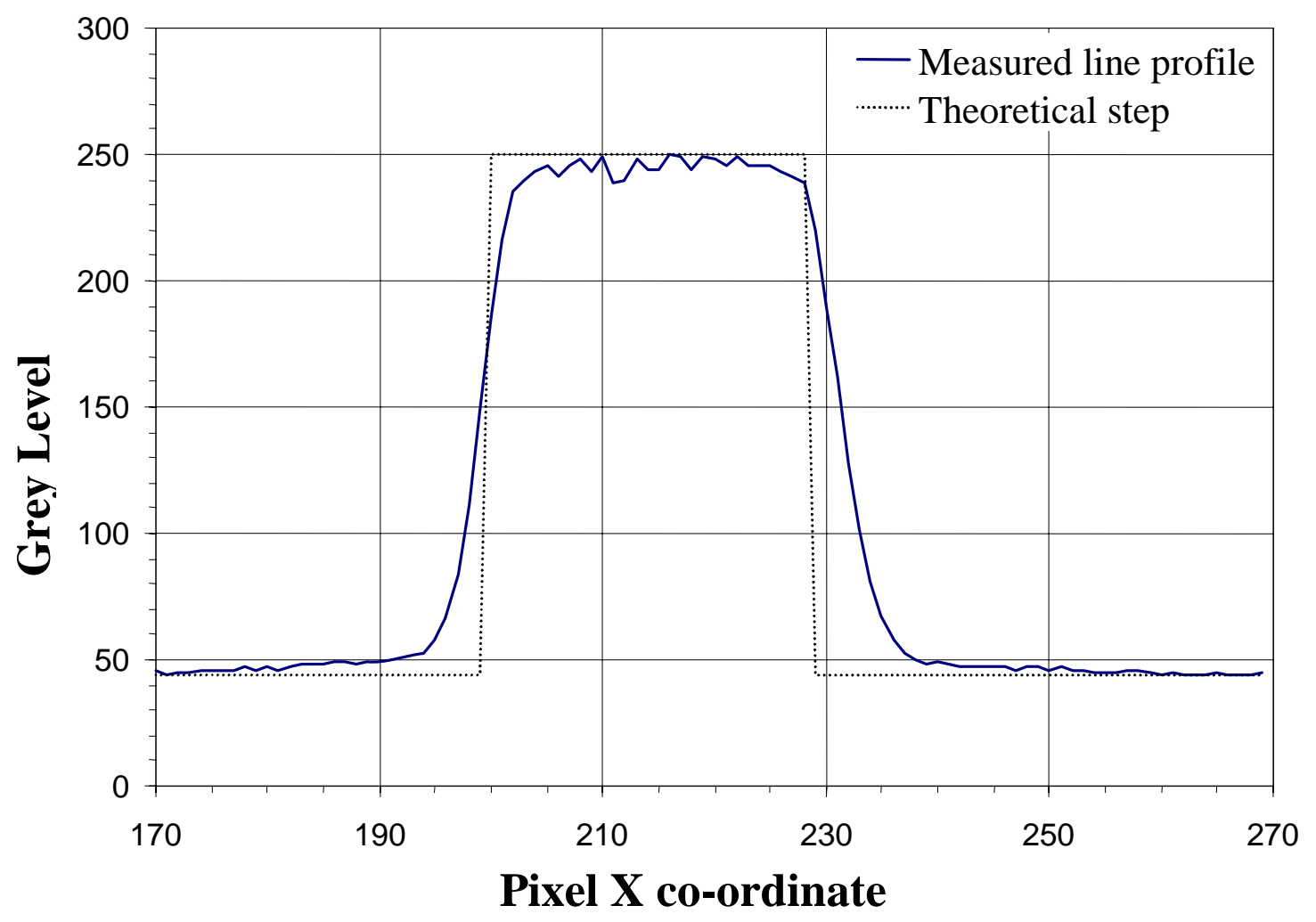

Fig. 12. Illuminated zones' line profile compared to a theoretical step for an incidence normal to the screen surface $\left(\theta_{1}=\Theta_{0}\right)$. 


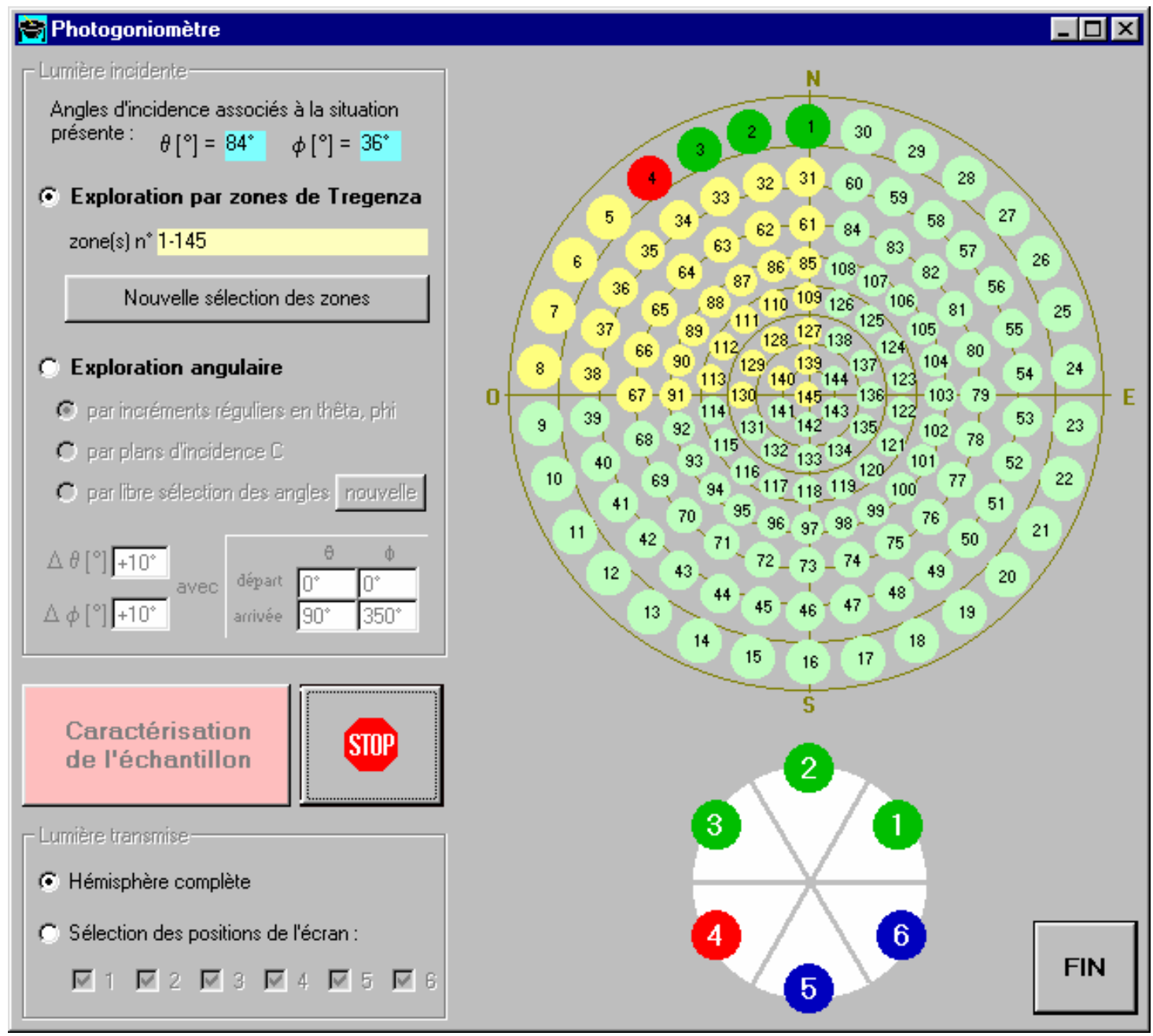

Fig. 13. Driving software interface for sample characterisations. There are mainly three ways of exploring the incident hemisphere (Tregenza distribution, steps in altitude along $C$ planes, free or regular angular steps), combined to a graphical illustration of the characterisation's evolution, with symmetries taken into account. 

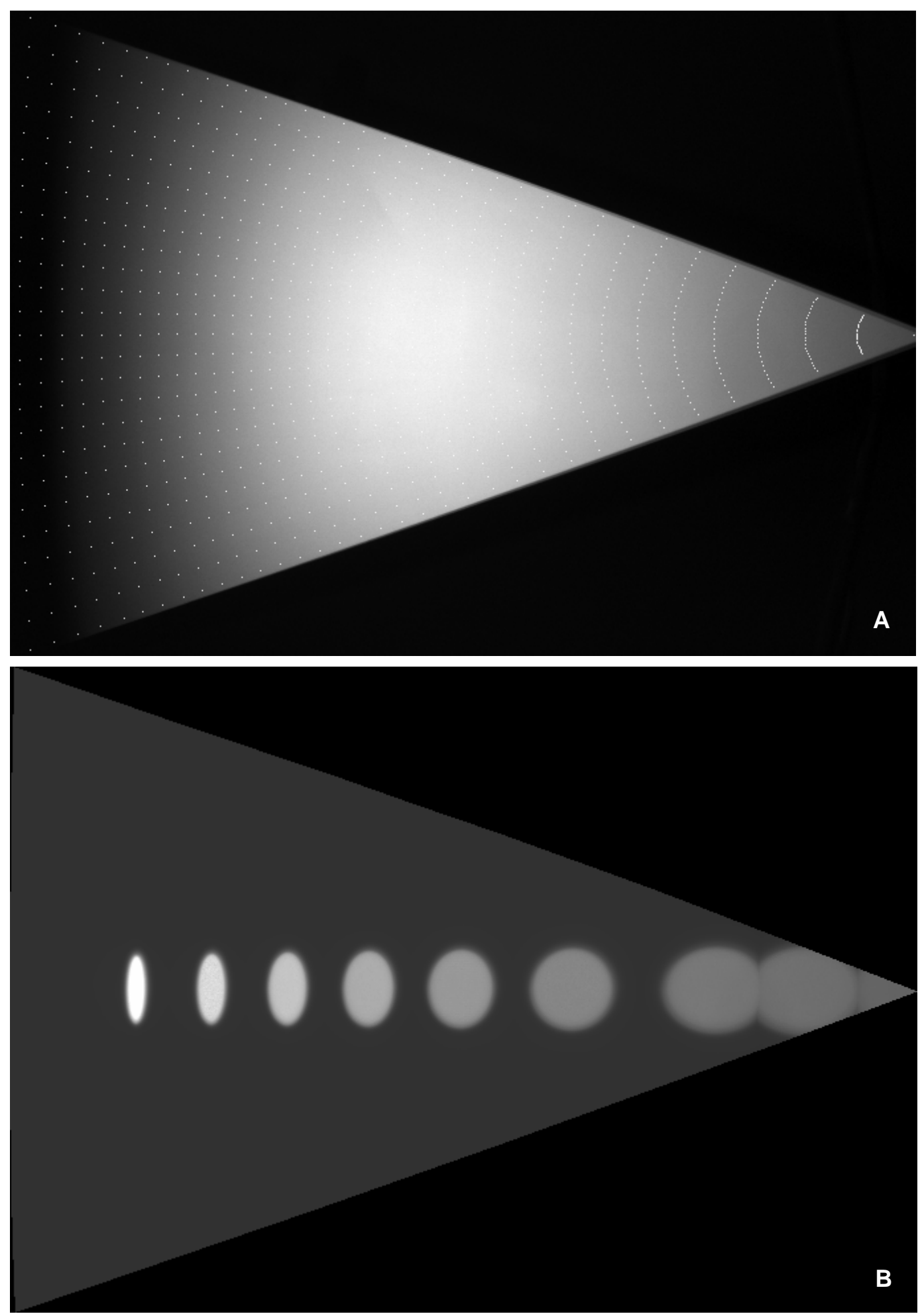

Fig. 14. A Lambertian diffuser. Points of equal altitude $\theta_{2}$ are expected to be of equal luminance, once their distance and light tilting effects have been compensated. $\mathbf{B}$ Superposition of images obtained without any sample (hole). The centre pixels inside each illuminated zone are expected to have values deduced from relation (5). 


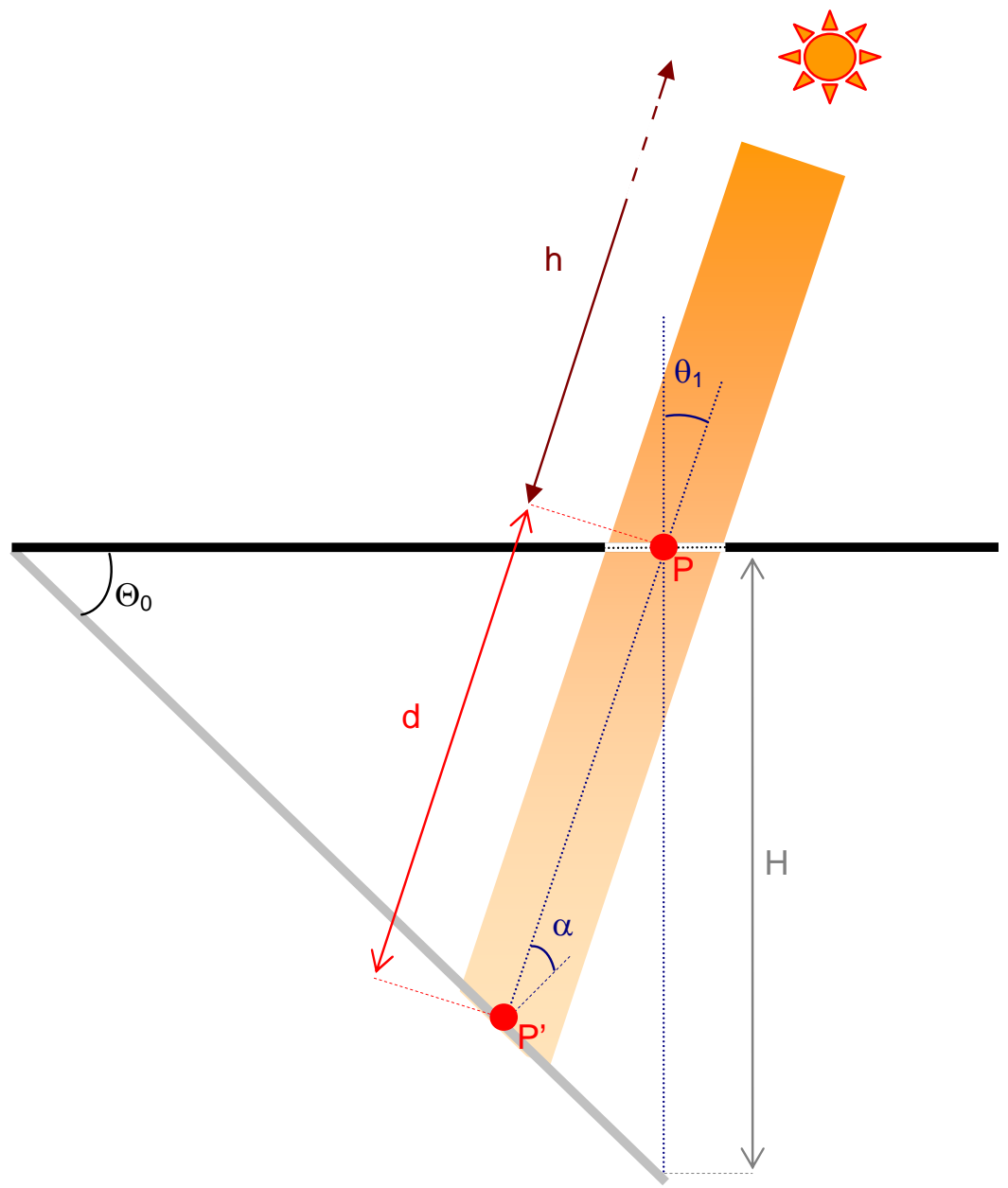

Fig. 15. Geometric characteristics of an incident beam reaching the screen without any sample. 


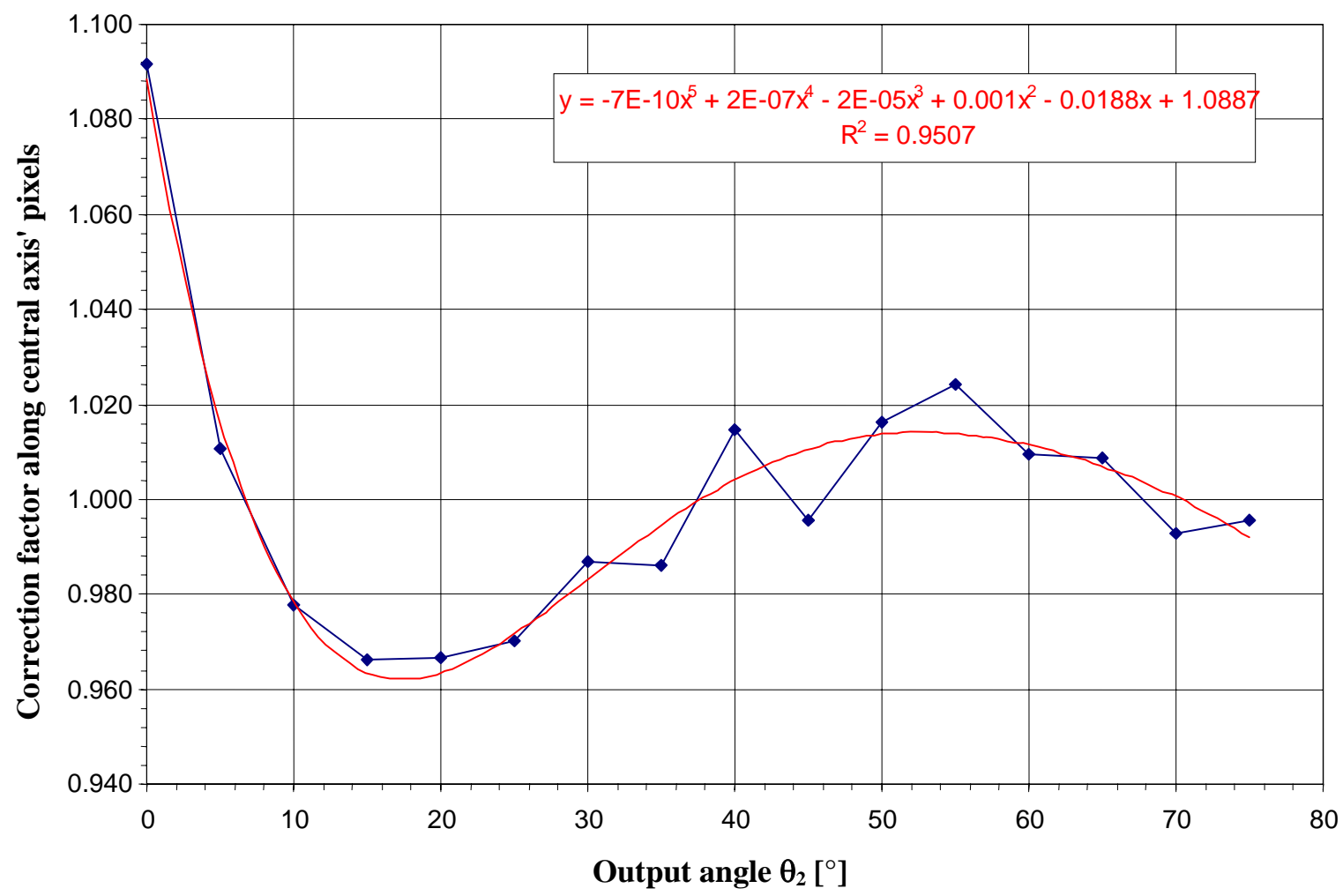

Fig. 16. Correction factor evolution along central screen axis. 


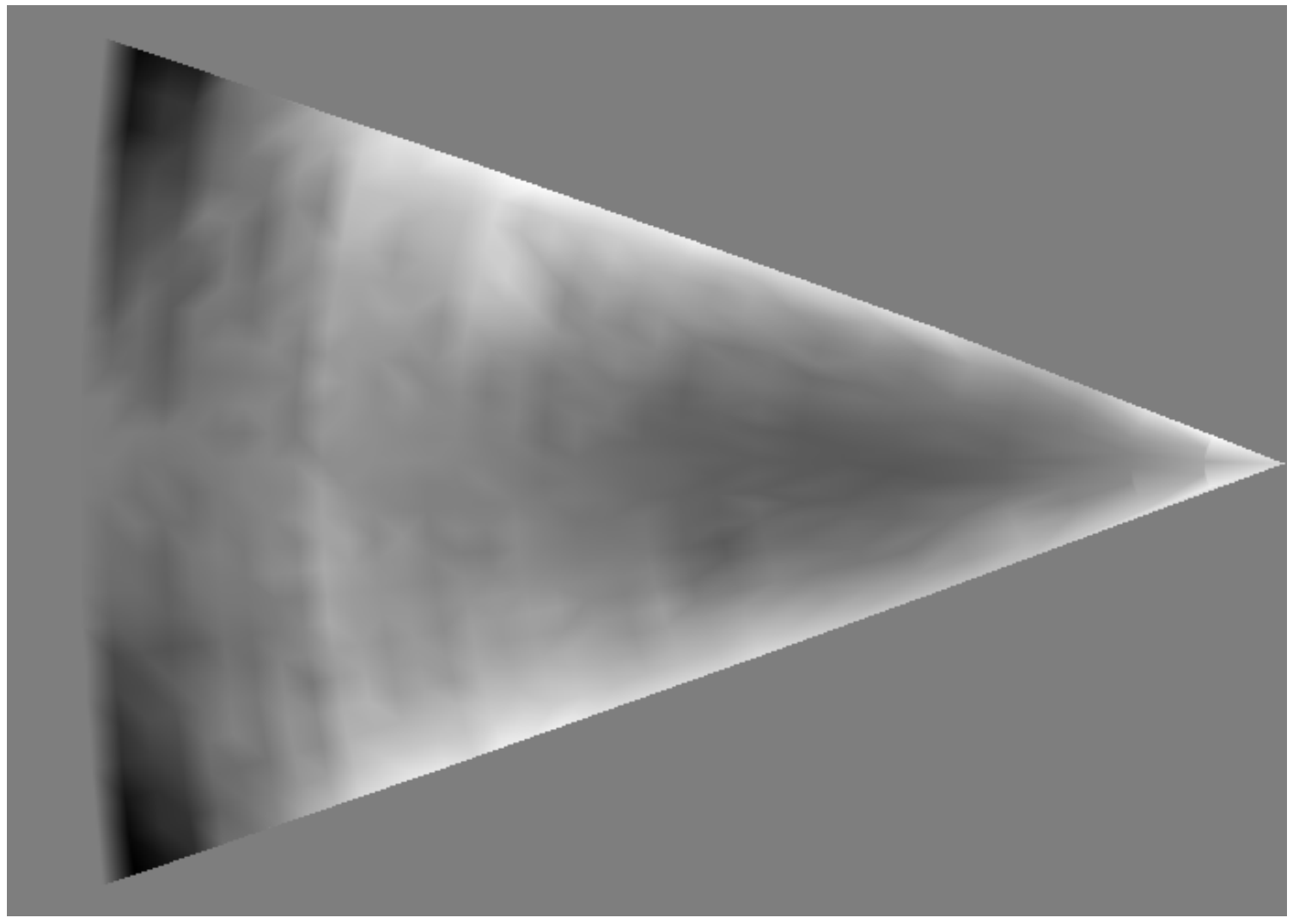

Fig. 17. Correction figure for image non-uniformity and imperfect diffusion of triangular screen: grey levels are to be associated with correction factor values: 0.88 (black) to 1.13 (white) ; the image region outside the measured area (screen) is arbitrarily fixed to 1. 

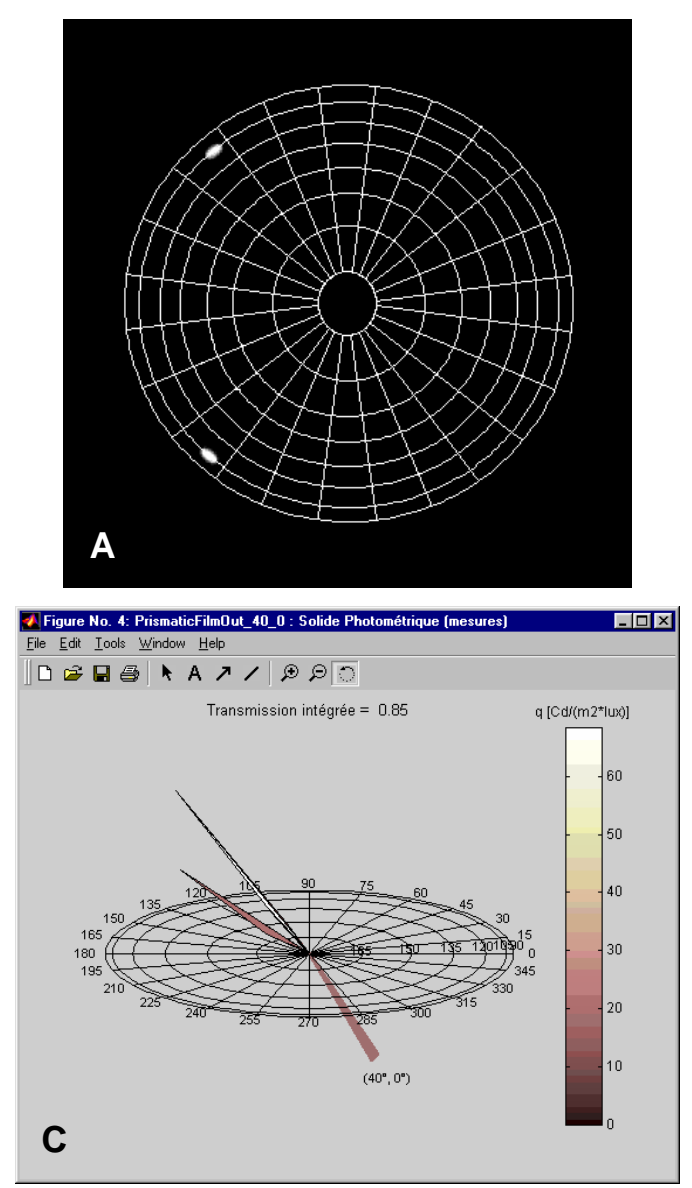
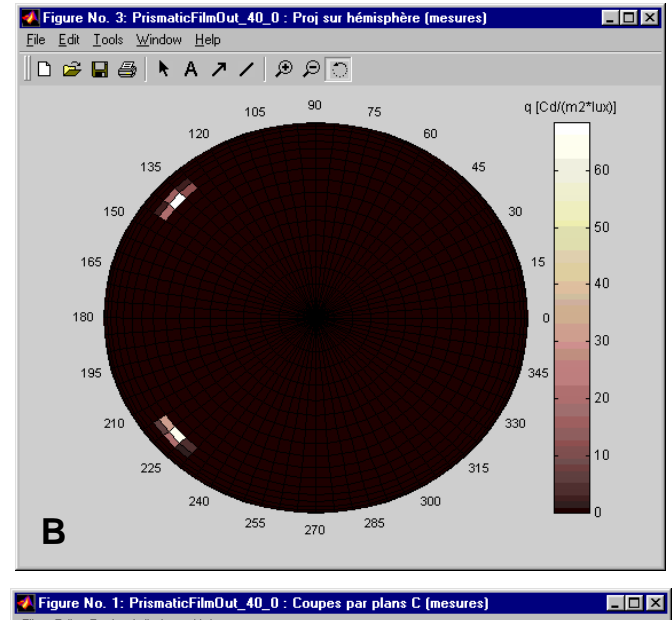

AFigure No. 1: PrismaticFilm0ut_40_0 : Coupes par plans $\mathrm{C}$ (mesures] Lools Window $\underline{H}$ elp

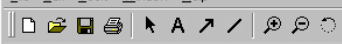

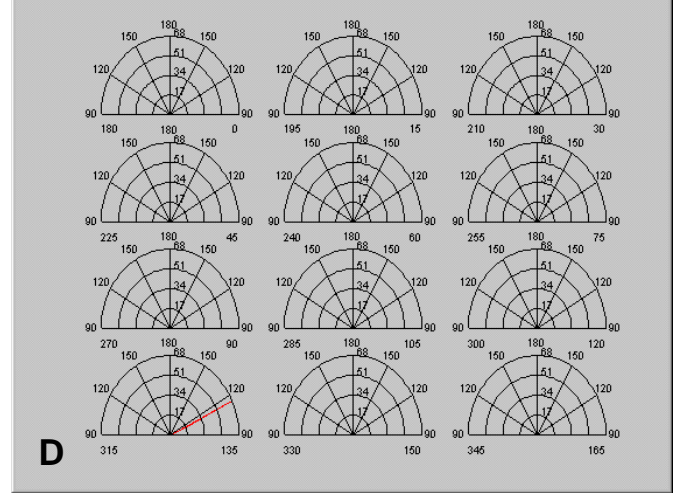

Fig. 18. Prismatic SOLF film (3M) : light incidence on prismatic side; $\left(\theta_{1}, \phi_{1}\right)=\left(40^{\circ}, 0^{\circ}\right) ; \varnothing=$ $10 \mathrm{~cm} ;\left(\Delta \theta_{2}, \Delta \phi_{2}\right)=\left(5^{\circ}, 5^{\circ}\right)$. A Calibrated image recomposed from sixths. B Hemispherical projection. C Photometric solid. D Section views along $C$ planes. 

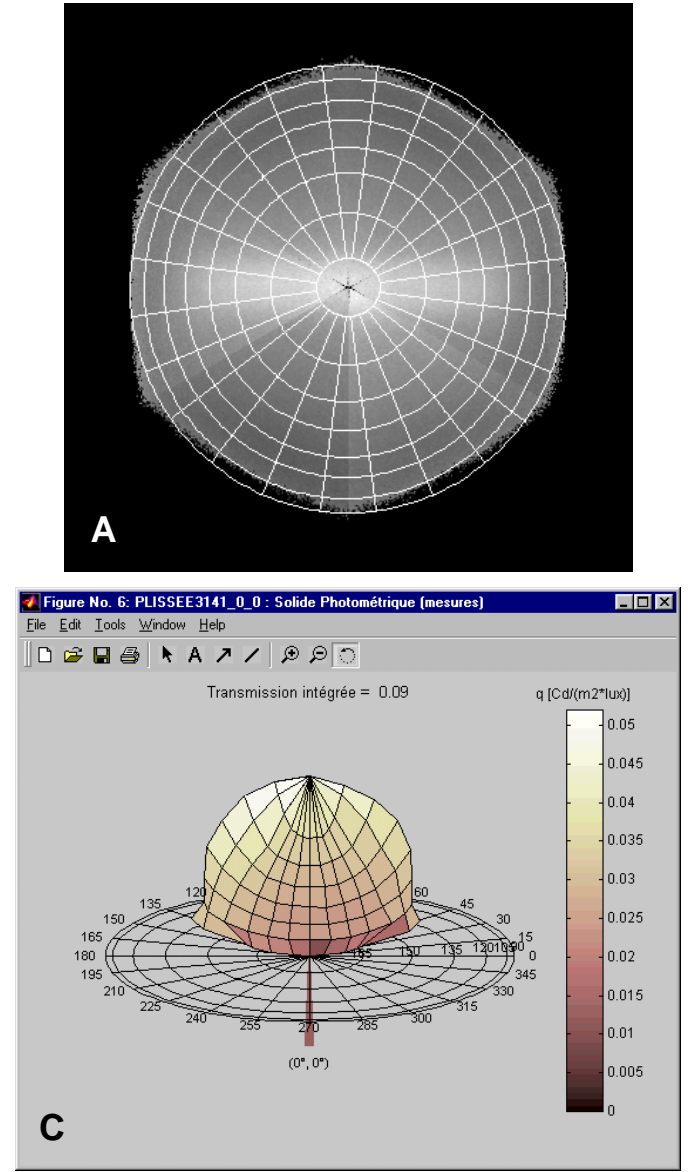
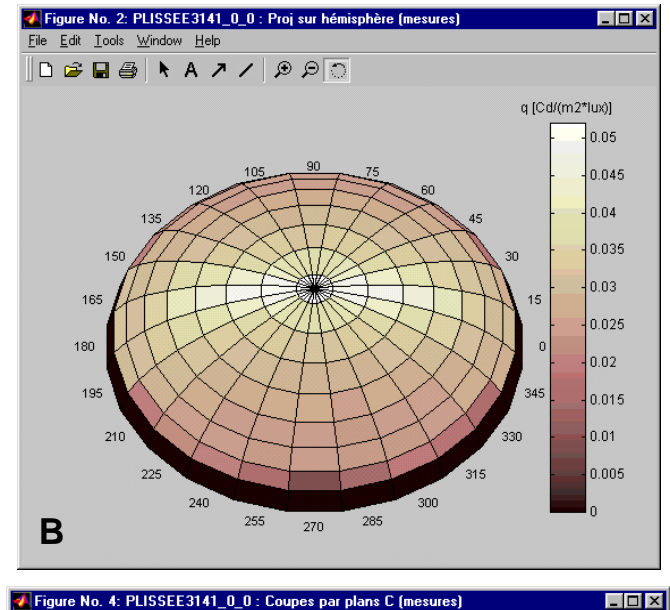

File Edit Iols Wussew His

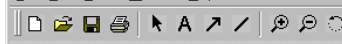

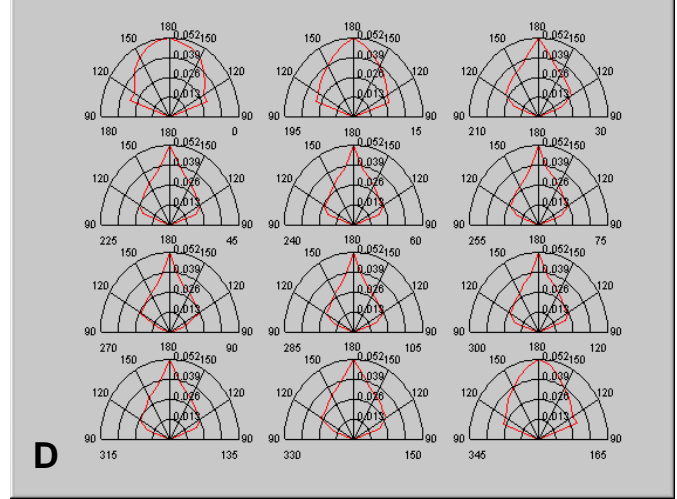

Fig. 19. Pleated textile solar blind (Baumann-Hüppe AG) : $\left(\theta_{1}, \phi_{1}\right)=\left(0^{\circ}, 0^{\circ}\right) ; \varnothing=17 \mathrm{~cm} ;\left(\Delta \theta_{2}\right.$, $\left.\Delta \phi_{2}\right)=\left(10^{\circ}, 15^{\circ}\right)$. A Calibrated image recomposed from sixths. B Hemispherical projection. C Photometric solid. D Section views along C planes. 


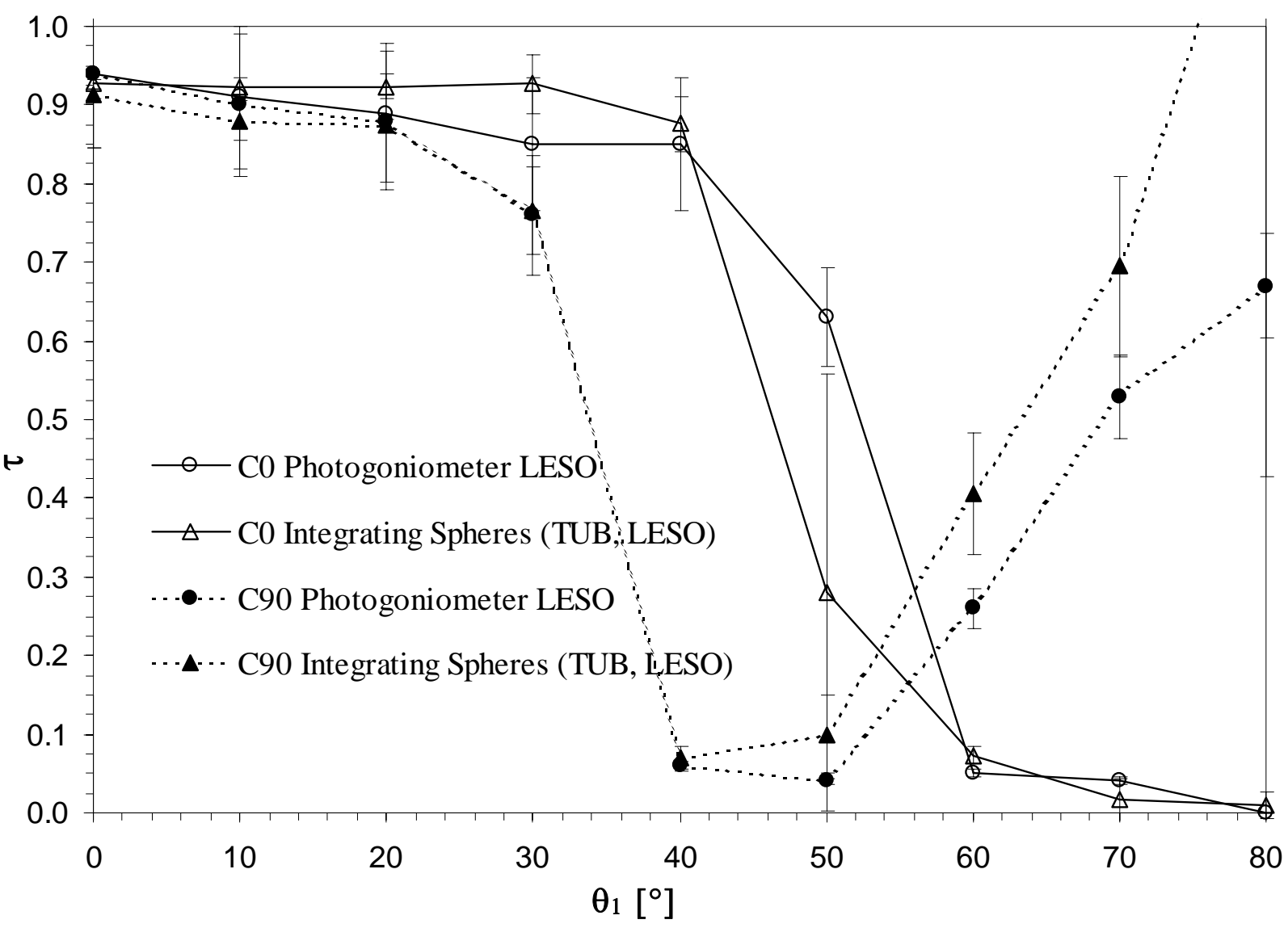

Fig. 20. Integrated light transmittance of prismatic SOLF film (3M), with light incidence on prismatic side. The Integrating Spheres results are based on an average value obtained from measurements of the same samples in different laboratories, with a standard deviation given by the error bars. 